\title{
Service Region Design for Urban Electric Vehicle Sharing Systems
}

\author{
Long He \\ NUS Business School, National University of Singapore, Singapore, longhe@nus.edu.sg \\ Ho-Yin Mak \\ Saïd Business School, University of Oxford, United Kingdom, ho-yin.mak@sbs.ox.ac.uk \\ Ying Rong \\ Antai College of Economics and Management, Shanghai Jiao Tong University, Shanghai, China, yrong@sjtu.edu.cn
}

Zuo-Jun Max Shen

Department of Industrial Engineering and Operations Research and Department of Civil and Environmental Engineering, University of California, Berkeley CA, USA, maxshen@berkeley.edu

Emerging collaborative consumption business models have shown promise in terms of both generating business opportunities and enhancing the efficient use of resources. In the transportation domain, car sharing models are being adopted on a mass scale in major metropolitan areas worldwide. This mode of servicized mobility bridges the resource efficiency of public transit and the flexibility of personal transportation. Beyond the significant potential to reduce car ownership, car sharing shows promise in supporting the adoption of fuel- efficient vehicles, such as electric vehicles (EVs), due to these vehicles special cost structure with high purchase but low operating costs. Recently, key players in the car sharing business, such as Autolib, Car2Go and DriveNow, have begun to employ EVs in an operations model that accommodates one-way trips. On the one hand (and particularly in free-floating car sharing), the one-way model results in significant improvements in coverage of travel needs and therefore in adoption potential compared with the conventional round-trip-only model (advocated by ZipCar, for example). On the other hand, this model poses tremendous planning and operational challenges. In this work, we study the planning problem faced by service providers in designing a geographical service region in which to operate the service. This decision entails trade-offs between maximizing customer catchment by covering travel needs and controlling fleet operations costs. We develop a mathematical programming model that incorporates details of both customer adoption behavior and fleet management (including EV repositioning and charging) under imbalanced travel patterns. To address inherent planning uncertainty with regard to adoption patterns, we employ a distributionally robust optimization framework that informs robust decisions to overcome possible ambiguity (or lacking) of data. Mathematically, the problem can be approximated by a mixed integer second-order cone program, which is computationally tractable with practical scale data. Applying this approach to the case of Car2Go's service with real operations data, we address a number of planning questions and suggest that there is potential for the future development of this service.

Key words: sustainable operations; car sharing; electric vehicles; robust optimization ; facility location History: This paper is accepted for publication in Manufacturing \& Service Operations Management in October 2016. 


\section{Introduction}

The emerging sharing economy enables novel business models that aim to improve resource utilization. From houses (Airbnb), to cars (ZipCar, Uber), to manpower for small tasks (TaskRabbit) and even dog kennels (DogVacay), innovative business models allow users to access and share resources without owning them. It is currently estimated that the sharing economy market is worth $\$ 26$ billion (The Economist 2013). Passenger cars make a prime candidate for sharing business models because of their low utilization rates (idle $92 \%$ of the time, on average (Atcheson and Green 2012)), high fixed costs to own ( $\$ 6,500$ per year, on average) and relatively low variable costs to operate. Worldwide, the number of car sharing users is forecast to grow from 2.3 million in 2013 to 12 million in 2020 (Reuters 2013). The largest car sharing firms, Car2Go and ZipCar, operate in multiple cities with total fleet sizes of over 10,000 each. The potential for improving efficiency is clear from reduced car ownership - households are found to own 0.23 fewer cars, on average, after adopting car sharing (ACCESS 2011).

Car sharing has also been proposed as a viable solution to sustainable transportation development, a topic drawing increasing attention in recent years as public awareness of environmental issues has grown. In 2012, the transportation sector accounted for $28 \%$ of total U.S. greenhouse gas (GHG) emissions (U.S. Environmental Protection Agency 2014). Moreover, approximately $70 \%$ of U.S. oil consumption can be attributed to transportation activities (Center for Climate and Energy Solutions 2014). Adoption of car models with better fuel economy is a key factor in curbing GHG emissions. Car sharing fleets are found to be more fuel efficient by 10 miles per gallon, on average, than vehicles owned by households (ACCESS 2011). Because fuel-efficient cars are typically more expensive to purchase and cheaper to operate (due to lower fuel costs), they are natural candidates for car sharing operations.

To improve fuel economy, innovative alternative fuel vehicles, including electric vehicles $(\mathrm{EVs})$, have been commercialized by auto manufacturers as alternatives to conventional internal combustion engine (ICE) vehicles. EVs have no tailpipe emissions, and they offer significant improvements on well-to-wheel energy efficiency and emissions levels over their ICE counterparts, when powered by efficient and more diverse sources of electricity (e.g., solar and wind power). The diversity of power sources also makes EVs less sensitive to fossil fuel depletion and to supply uncertainty of crude oil. From the consumer's perspective, 
EVs boast low operational costs: the fuel cost per mile for passenger EVs is approximately 4 cents in the U.S., compared with 12 cents for average ICE vehicles (U.S. Department of Energy 2014b). Despite their potential, consumers are not ready to own EVs on a massive scale due to several major hurdles, including their short driving ranges (coupled with insufficient numbers and inadequate distribution of charging facilities), their high upfront purchase cost and their potentially high depreciation rate due to rapid technology development.

Notably, the combination of EVs with car sharing operations is emerging globally as a viable alternative to EV ownership for urban dwellers. Car2Go, a subsidiary of Daimler AG, operates a car sharing system with full EV fleets in San Diego (U.S.), Amsterdam (Netherlands) and Stuttgart (Germany). DriveNow (rebranded as ReachNow in the U.S.), operated by BMW, serves Copenhagen with all EVs (DriveNow 2016) and provides mixed fleets of EVs and ICEs in several cities, including Seattle (U.S.), London (U.K.), Berlin and Munich (Germany) (ReachNow 2016). Autolib has deployed over 2,000 EVs in Paris (France) through its EV sharing service (Autolib 2016). This innovative operations model can potentially help overcome the major barriers to EV adoption for a number of reasons. First, concerns about EVs range limitation in sharing systems are alleviated because they operate almost exclusively in well-defined urban service areas. The concentration of a sizable fleet within a dense urban area also makes the development of a charging infrastructure more feasible. Second, car sharing effectively allows a pool of users to amortize the high fixed costs of purchasing EVs (and maintaining them) into usage-based variable costs over their collective consumption of the service. By pooling consumers driving needs, having EVs in sharing fleets results in higher utilization, and average costs can thus be reduced compared with the case of individual ownership. As a consequence of higher utilization, the environmental benefits yielded by each EV also increases over the case of individual ownership. Third, by retaining ownership, the firm effectively eases consumers' concerns about technological risks, future resale values, and maintenance.

In addition to introducing EVs to car sharing, Autolib, Car2Go and DriveNow differ from early station-based car sharing systems, such as Zipcar, by allowing both round trips and one-way trips in their free-floating car sharing systems. Specifically, Car2Go and DriveNow allow customers to check out and return cars anywhere within the service region at any street parking space, whereas Autolib allows customers to check out from 
and return to designated parking spots. This flexibility allows customers to use the service for regular trips with long stopover times (e.g., commuting to office or school), which are typically not economically feasible under round-trip only services. Figure 1 shows the frequencies of trips classified by origin-destination distances, obtained from Car2Go's onemonth operational data in San Diego. The destination is defined as the location at which a rental trip is ended. We observe that in the majority of trips, origin-destination distances extend beyond walking distance (e.g., $\geq 2 \mathrm{~km}$ ) and a substantial proportion may not be served when round-trips are required (e.g., in ZipCar's system).

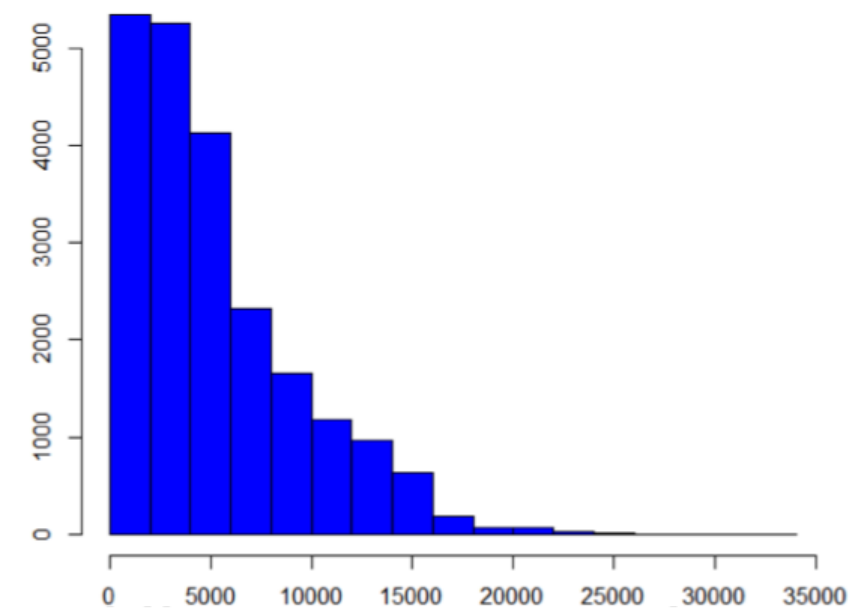

Figure 1 Histogram of the Origin-Destination Distances (in meters) of One-month Car2Go Trips

Although allowing one-way trips opens up a broader potential customer base, it makes fleet operations more difficult. Thus, a key strategic planning task in free-floating car sharing systems is to determine the service region. On the one hand, expanding geographical coverage entails significant operational challenges, such as the repositioning of cars to ensure availability under imbalanced demand and scheduling for recharging in the case of EVs. On the other hand, customer adoption is ultimately dependent on service coverage, as travel needs can only be covered when both the trip origin and destination are within the service region. Hence, coverage of a more extensive service region encourages adoption and improves potential revenue.

In this paper, we address the strategic planning problem of service region design for one-way EV sharing systems, which entails several challenges. First, the travel pattern and adoption behavior of potential customers are highly uncertain at the planning stage. 
Moreover, before entering a new city or considering a major service expansion, the firm may not possess accurate data to describe the uncertainty in terms of probability distributions, which exacerbates the planning challenge. Because strategic commitments (such as the acquisition of land for stations and charging outlets) are often made in conjunction with service region design, a robust planning methodology is imperative. Second, the operational details of EV sharing, such as repositioning and recharging of EVs, depend on both the size and shape of the service region. Hence, the firm must also conscientiously account for operational cost drivers when determining the service region when only limited data are available. In this paper, we model the service region design problem by taking these factors into account. Our contributions can be summarized as follows.

- We formulate an integrated service region planning model that considers customers' satisficing behavior in service adoption together with various operational characteristics of a one-way EV sharing system. Our approach deliberately addresses data uncertainty and ambiguity with regard to customers' travel patterns. Using a distributionally robust optimization framework, our model can be approximated by a computationally efficient mixed integer second-order cone program (MISOCP).

- Using real operations data from Car2Go, data regarding travel characteristics from the California Household Travel Survey and EV charging station deployment data from the U.S. Department of Energy, we perform a case study of Car2Go's service region design in San Diego. We address several planning questions, and we make the following findings.

1. EV sharing systems deliver more environmental benefits, such as savings in $\mathrm{CO}_{2}$ emissions, than replacing individually owned gasoline cars with EVs.

2. While faster charging technologies help enhance profit and service coverage by improving fleet utilization, the benefits diminish as charging speed improves. Thus, it is sufficient for the car sharing system to deploy moderately fast, but not necessarily the fastest, charging equipment.

3. The optimal service region tends to be larger when customers' valuation of the availability of cars is lower and when customers' valuation of service coverage is higher.

The remainder of this paper is organized as follows. In Section 2, we review several related streams in the literature. In Section 3, we first present our model, which considers both the customer adoption and fleet operations aspects of the EV sharing system. Next, in Section 4, we demonstrate the model using a case study with real data and discuss our findings based on the computational results. Finally, we conclude the paper in Section 5. 


\section{Literature Review}

Our work contributes to the expanding research on sustainable operations management, which covers a wide range of topics (Kleindorfer et al. 2005, Plambeck 2013). A stream in the literature addresses sustainable operations related to EV business models. Lim et al. (2015) evaluate business practices aiming at the goal of mass adoption by studying the impact of range and resale anxieties. Similarly, Avci et al. (2014) highlight the key mechanisms driving the adoption and use of EVs in a battery swapping system. In particular, the build a behavioral model of motorist use and adoption; calibrating their model to real data, they find that such a system may not always be beneficial to the environment. In addition to the insights from their business model analysis, they also study infrastructure planning and charging coordination issues. Mak et al. (2013) develop distributionally robust optimization models that assist in the planning process for deploying battery swapping infrastructure for EVs. Moreover, a few papers in transportation optimize the operations of charging station networks and coordinate recharging schemes by means of area pricing or routing (Flath et al. 2013, Schneider et al. 2014). In the present study, we consider EV charging operations together with customer adoption of the service instead of EV ownership all in the context of an EV sharing system.

Although there are several major hurdles to achieving mass EV adoption, EV sharing is an alternative for customers to enjoy the benefits of EVs without ownership. There are several prevalent shared transport service models, such as peer-to-peer sharing (e.g., Turo), ride sharing (e.g., Uber) and car sharing (e.g., Car2Go), in the emerging sharing economy. A number of papers have studied various peer-to-peer platforms where customers decide to buy or rent the products (Benjaafar et al. 2015, Fraiberger and Sundararajan 2015, Jiang and Tian 2016). Recent developments of ride-sharing platforms have also motivated research in the matching mechanism, wage and price of on-demand services (Banerjee et al. 2015, Cachon et al. 2015, Hu and Zhou 2016, Tang et al. 2016, Taylor 2016). In an EV sharing system, the high fixed costs of EV ownership are transformed into a usage-based cost of service. Researchers have used the term servicizing to describe a business model that offers the functionality of the products instead of selling the product itself. Agrawal and Bellos (2016) assess the potential of servicizing business models as an environmentally sustainable strategy and draw insights into when and how servicizing is environmentally beneficial. Related to this study, Bellos et al. (2015) determine an OEM's optimal pricing strategy 
and the optimal fleet size when it offers car sharing in conjunction with conventional sales, revealing the discrepancy between profitability and environmental sustainability. Because the car-sharing system in their model allows for only round trips, they can focus on the fleet operations of each station individually as a single server, whereas in our model, the EV sharing system is designed to support one-way trips, and fleet repositioning is necessary to accommodate imbalanced trip flows.

One-way vehicle sharing systems are also studied by both the operations management and the transportation communities. Using high-frequency system-use data from a bicycle sharing system, Kabra et al. (2015) measure the accessibility of stations and the availability of bicycles. They then estimate the effects of accessibility and availability on ridership through a novel transformation technique and reveal a potential opportunity for service improvement in Paris using a calibrated counterfactual simulation. Several works from the transportation domain investigate planning and vehicle relocation problems in oneway station-based car-sharing systems, e.g., (Bruglieri et al. 2014, de Almeida Correia and Antunes 2012, Nourinejad et al. 2015, Febbraro et al. 2012, Boyacı et al. 2015), and freefloating car sharing systems, e.g., (Weikl and Bogenberger 2013). However, the main focus of these models is on operations, such as the optimization of repositioning schedules under known demand patterns (or scenarios). By contrast, we focus on the strategic decisions of service region design and consider demand adoption uncertainty as well as the fleet operations issues, including repositioning and time-varying demand patterns.

Shu et al. (2013) consider detailed bicycle sharing operations in a network context. Whereas their work focuses on operational admission control, we consider strategic region design with consumer adoption behavior. Shu et al. (2013) develop a network flow model with proportionality constraints to characterize the flow of bicycles within the network and the number of trips supported. Using transit data from the transit rail operator in Singapore, they examine bicycles deployment, utilization, and (the value of) redistribution. Due to bicycles short range, they restrict their consideration to trips within two transit stops and assume that bicycles are immediately available for the next customers upon arrival. However, in EV sharing systems, there is a risk that the arriving EVs will have a low battery level and must be placed out for recharging. Moreover, our work models the repositioning of the fleet as a stochastic process, whereas Shu et al. (2013) consider a system that restores the bicycles distribution among all locations on a regular basis. 
Furthermore, their model assumes that the bicycle sharing station locations are given and that the demands follow known Poisson processes. In our study, we aim to design a service region under incomplete information regarding consumer adoption and travel behaviors.

Because service providers must determine the service region before the system is in operation and before customers sign up for membership, demand uncertainty is a major concern. It is therefore critical to obtain a service region design that is robust with regard to various adoption scenarios. The literature on robust optimization (Ben-Tal and Nemirovski 1998, 1999, Bertsimas and Sim 2003, 2004) has developed approaches to prescribe solutions that are robust with respect to perturbations in model parameters. For problems in which some limited distributional information (such as the means and covariances of key parameters) may be available at the planning stage, it is possible to utilize the distributionally robust optimization approaches discussed in El Ghaoui et al. (2003), Chen et al. (2007, 2010), Goh and Sim (2010), Delage and Ye (2010) and Natarajan et al. (2011). An advantage of this methodology is that it is often possible to preserve computational tractability using conic programming formulations. A recent application in EV infrastructure planning can be found in Mak et al. (2013). With some limited information, such as the moments of demand parameters, they develop distributionally robust models for deployment with EV battery swapping stations. Their formulations are tightly approximated by mixed integer second-order cone programs (MISOCPs), which are readily solvable by commercial solvers. Other recent applications include appointment scheduling in healthcare (Kong et al. 2013, Mak et al. 2014), warehouse operations (Ang et al. 2012), supply chain management (Mak and Shen 2014), inventory control (See and Sim 2010) and portfolio management (Natarajan et al. 2010).

\section{The Model}

We consider an urban EV sharing service provider (or the firm) that designs its service region to serve a metropolitan area. A one-way free-floating car sharing service is typically operated within a well-defined service region. An overview of the current service region of Car2Go San Diego is shown in Figure 2. A feature that differentiates free-floating car sharing systems from station-based systems is that one-way trips and free street parking are allowed in the former. Customers can begin trips anywhere inside the service region and can end trips wherever there are legal parking spaces (for detailed parking rules, please 
refer to (Car2Go 2016)). Moreover, customers can drive and make stopovers outside the service region during reservations but are required to return the car to any point within the service region by the end of a trip (Car2Go 2016).

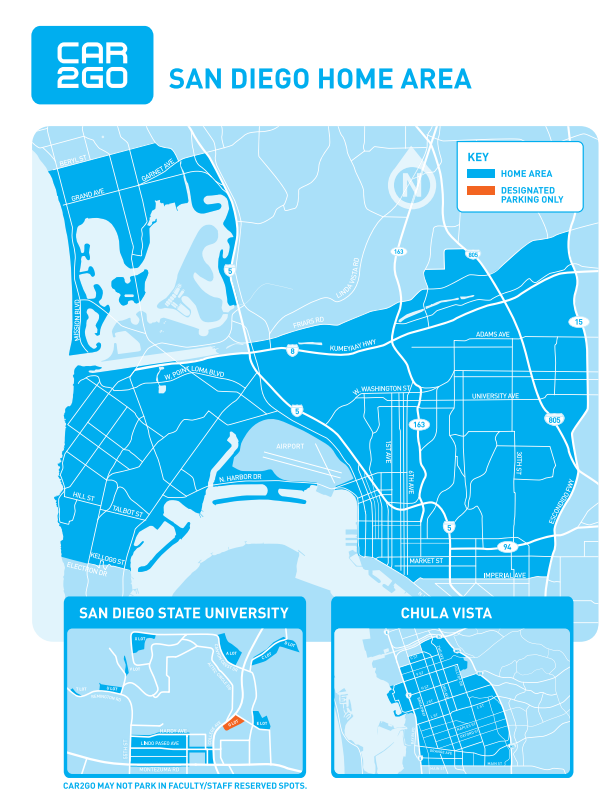

Figure 2 Service Region of Car2Go San Diego as of February 2015 (Car2Go 2015)

Due to the free-floating nature of the service, a well-planned service region balances the goals of encouraging customers to adopt the service and maintaining cost-effective fleet operations. From the customers' perspective, it is more favorable to adopt and use an EV sharing service if the service region covers more of their preferred destinations. Nevertheless, a larger service region may result in more complex operations and higher operational costs to the service provider, as a result. Hence, it is crucial to model the interrelationships among customer adoption, fleet operations, and service region design. However, it is difficult in practice to obtain accurate estimations of individual valuations on destination coverage. In the model, we attempt to depict the aggregate customer adoption levels of the EV sharing service, and we propose an optimization model that strategically supports service region design under uncertainty of customer travel patterns and preferences.

We consider an urban area consisting of a set $I$ of non-overlapping geographical locations (e.g., districts). The firm designs its service region by selecting a subset of $I$ to cover. Mathematically, we define binary decision variables $x_{i}$ for each $i \in I$ to indicate whether 
location $j$ is covered in the service region $\left(x_{i}=1\right)$ or not $\left(x_{i}=0\right)$. Throughout this paper, unless otherwise specified, we use boldface letters to denote matrices or vectors consisting of scalar parameters or variables denoted by the same letter. Thus, $\mathbf{x}$ is the vector whose components are $x_{i}$ for $i \in I$. We also define the inner product $\langle A, B\rangle=\operatorname{trace}(\mathbf{B A})$ for two matrices $\mathbf{A}$ and $\mathbf{B}$.

We consider the following satisficing behavior of service adoption. Let $a_{i j}$ represent the utility that a customer who resides in location $i$ potentially derives from being able to travel from $i$ to $j$, which depends on both the frequency of making these trips and the value of such trips to the customer. Therefore, as long as the origin $i$ is covered by the service, the customer could enjoy a potential utility of $\sum_{j \in I} a_{i j} x_{j}$ by adopting the service. Under the satisficing behavior in service adoption (e.g., Simon 1957), the customer would adopt the service when the total utility from the service coverage of the destinations exceeds his or her aspirational level. Customers are categorized into $K$ groups and the customers in group $k$ have the same aspirational level $b_{k}$. Thus, given that the origin $i$ is served, the adoption decision of a customer in group $k$ can be described by the following indicator function:

$$
\mathbf{1}\left(\sum_{j \in I} a_{i j} x_{j} \geq b_{k}\right)=\left\{\begin{array}{lr}
1, & \text { if } \sum_{j \in I} a_{i j} x_{j} \geq b_{k} \\
0, & \text { otherwise }
\end{array}\right.
$$

Let $a_{i j}^{n}$ be the utility value associated with an $i-j$ trip for a customer indexed by $n$. Naturally, the utility values are heterogeneous among individual customers. From the planner's perspective, one may view the values of $a_{i j}^{n}$ as independent realizations drawn from a probabilistic distribution $p$ of a random variable $a_{i j}$. Therefore, we consider the adoption rate at the aggregate level, defined as the probability of customer adoption. By taking expectation over $a_{i j}$ in the indicator function, the adoption rate $q_{i k}$ is given by:

$$
\begin{aligned}
q_{i k} & =\mathbb{E}\left[\mathbf{1}\left(\sum_{j \in I} a_{i j} x_{j} \geq b_{k}\right)\right] \\
& =\operatorname{Prob}\left(\sum_{j \in I} a_{i j} x_{j} \geq b_{k}\right) .
\end{aligned}
$$

The firm's profit consists of membership revenue and operational profit. Each customer who signs up for the service pays an annual membership fee $f$ and is then charged per unit time of usage. To serve region $i \in I$, the firm incurs a fixed cost, $g_{i}$, which may include 
investments in charging infrastructure or payments to charging service providers and city parking permit costs. Our model maximizes the expected total profit, which is defined as total revenue less fixed and operational costs (including charging cost, repositioning cost and fleet investment), as formulated in Equation (1) below. For notational brevity, operational profit is represented by a function $\Theta(\mathbf{q}, \mathbf{x})$ with service region $\mathbf{x}$ and realized adoption rates $\mathbf{q}$. We provide an explicit formulation for $\Theta(\cdot)$ in Section 3.2. The service region design problem is formulated as:

$$
\begin{aligned}
& \max _{\mathbf{q}, \mathbf{x}} \sum_{i \in I} \sum_{k \in K} f Q_{i k} q_{i k}-\sum_{i \in I} g_{i} x_{i}+\Theta(\mathbf{q}, \mathbf{x}) \\
& \text { s.t. } \\
& \qquad q_{i k} \leq \operatorname{Prob}\left(\sum_{j \in I} a_{i j} x_{j} \geq b_{k}\right), \forall i \in I, \forall k \in K \\
& \quad q_{i k} \leq x_{i}, \forall i \in I, \forall k \in K \\
& \quad x_{i} \in\{0,1\}, \forall i \in I .
\end{aligned}
$$

With adoption rates $q_{i k}$, the expected total membership fee revenue is given by $\sum_{i \in I} \sum_{k \in K} f Q_{i k} q_{i k}$, where $Q_{i k}$ is the size of customer group $k$ in region $i$. Constraint (2) expresses the adoption rate as a probability constraint. Furthermore, constraint (3) stipulates that no customers will adopt the service if their origins are not served. Appendix A summarizes the notation used throughout the paper.

\subsection{Adoption Rate Model}

In this section, we focus on addressing the probability constraint (2). To evaluate the exact adoption rate, the firm requires complete information on the joint distribution of a. However, in practice, perfect information is often unavailable when the problem is to be solved. Specifically, as the firm is in the planning stage in which only limited operations data may be available (e.g., from pilot studies or surveys), it is often difficult to fit the joint distribution of travel patterns with confidence. Furthermore, from the tractability standpoint, the term $\sum_{j \in I} a_{i j} x_{j}$ is difficult to evaluate in general because of the need to numerically compute convolutions, even when the distributions are known and the components of a are independent. To this end, it is practical to consider a model that features both distributional robustness and computational tractability under limited information. 
In particular, we relax the data requirement by assuming knowledge of only certain descriptive statistics of a, i.e., their means and covariance matrices. We construct a robust model that delivers the worst-case adoption rate, i.e., the lowest adoption rate among all possible distributions $\mathcal{P}$ of the utility parameters a with the given means and covariance matrices:

$$
q_{i k} \leq \inf _{p \in \mathcal{P}} \mathbb{E}_{p}\left[\mathbf{1}\left(\sum_{j \in I} a_{i j} x_{j} \geq b_{k}\right)\right]
$$

The utility parameter $a_{i j}$ is a nonnegative random variable, as utility would not be reduced by additional coverage. In the following, we use $\mathbf{a}_{i}=\left(a_{i j}\right)_{j \in I}$ to denote the random vector of random utility parameters associated with the origin $i$. We assume that the mean vector $\overline{\mathbf{a}}_{i}=\left(\bar{a}_{i j}\right)$ and covariance matrix $\boldsymbol{\Gamma}_{i}=\left[\operatorname{cov}\left(a_{i j_{1}}, a_{i j_{2}}\right)\right]$ of $\mathbf{a}_{i}$ are known for each location $i \in I$. Then, the second moment matrix $\Sigma_{i}$ is given by:

$$
\boldsymbol{\Sigma}_{i}:=\mathbb{E}\left[\begin{array}{c}
\mathbf{a}_{i} \\
1
\end{array}\right]\left[\begin{array}{c}
\mathbf{a}_{i} \\
1
\end{array}\right]^{T}=\left[\begin{array}{cc}
\mathbf{S}_{i} & \overline{\mathbf{a}}_{i} \\
\overline{\mathbf{a}}_{i}^{T} & 1
\end{array}\right], \text { where } \mathbf{S}_{i}:=\Gamma_{i}+\overline{\mathbf{a}}_{i} \overline{\mathbf{a}}_{i}^{T} .
$$

We assume that the covariance matrix is positive definite, i.e., $\boldsymbol{\Gamma}_{i} \succ 0$, which implies that the second moment matrix is also positive definite, i.e., $\boldsymbol{\Sigma}_{i} \succ 0$. With a given $\mathbf{x}$, the worstcase adoption rate can be obtained by solving a convex optimization formulation with copositive constraints, as shown in Lemma 1. In other words, certain constraints in the formulation take the form of requiring certain decision variable matrices to be in the cone of copositive matrices. A symmetric matrix $\mathbf{M}$ is said to be copositive (denoted $\mathbf{M} \succeq_{c o} 0$ ) if it satisfies $\mathbf{v}^{T} \mathbf{M v} \geq 0, \forall \mathbf{v} \in \mathbb{R}_{+}^{n}$. For more details on copositive matrices and copositive programming, please refer to Burer (2009).

Constraint (2) in Problem (1) can be approximated by constraint (4). Furthermore, given the mean vector $\overline{\mathbf{a}}_{i}$ and the covariance matrix $\boldsymbol{\Gamma}_{i}$ for each region $i \in I$, we obtain the following result.

LEMMA 1. Under a service region design indicated by $\mathbf{x}$, the worst-case adoption rate constraint (4) is equivalent to the following set of inequalities with copositive constraints.

$$
\begin{aligned}
& \left\langle\mathbf{M}_{i k}, \boldsymbol{\Sigma}_{i}\right\rangle \leq 1-q_{i k} \\
& \mathbf{M}_{i k} \succeq_{c o} 0
\end{aligned}
$$




$$
\begin{aligned}
& \mathbf{M}_{i k}+\left[\begin{array}{cc}
0 & \mathbf{d}_{i k} \\
\mathbf{d}_{i k}^{T}-1-2 \tau_{i k} b_{k}
\end{array}\right] \succeq_{c o} 0 \\
& -\rho \mathbf{x} \leq \mathbf{d}_{i k} \\
& \mathbf{d}_{i k} \leq \rho \mathbf{x} \\
& \tau_{i k} \mathbf{e}+\rho(\mathbf{x}-\mathbf{e}) \leq \mathbf{d}_{i k} \\
& \mathbf{d}_{i k} \leq \tau_{i k} \mathbf{e}+\rho(\mathbf{e}-\mathbf{x}) \\
& \tau_{i k} \geq 0
\end{aligned}
$$

where $\rho$ is a large scalar and $\mathbf{e}$ is the vector of ones; $\mathbf{M}_{i k}$ (symmetric matrix), $\mathbf{d}_{i k}$ (vector), and $\tau_{i k}$ (scalar) are auxiliary decision variables.

Proof of Lemma 1. Please see Appendix B.1.

The formulation in Lemma 1 is not readily solvable by commercial solvers because of the combination of copositive constraints and integer-valued decision variables $\mathbf{x}$. A natural approach to addressing copositive constraints is to approximate them by tractable convex restrictions, particularly those involving a series of linear and semidefinite constraints. Moreover, we show that the semidefinite restriction of the copositive constraints in Lemma 1 can be further transformed into a second-order cone program. In Proposition 1, we provide a computationally tractable lower bound for the worst-case adoption rate in (4).

Proposition 1. Adoption rate $q_{i k}$ satisfies the following set of inequalities, with secondorder cone constraints (5), if and only if it satisfies Lemma 1 and constraint (4).

$$
\begin{aligned}
& \left\|\begin{array}{c}
1-q_{i k}-v_{i k} \\
2 \boldsymbol{\Gamma}_{i}^{\frac{1}{2}} \mathbf{x}
\end{array}\right\|_{2} \leq 1-q_{i k}+v_{i k}, \forall i \in I, \forall k \in K \\
& v_{i k}=b_{k}^{2}+\sum_{\left(j_{1}, j_{2}\right) \in I \times I}\left(\bar{a}_{i j_{1}} \bar{a}_{i j_{2}}+\sigma_{i j_{1} j_{2}}\right) z_{j_{1} j_{2}}-2 b_{k} \sum_{j \in I} \bar{a}_{i j} x_{j}, \forall i \in I, \forall k \in K \\
& \left(\mathbf{x}, q_{i k}\right) \in \mathcal{X}_{i k}, \forall i \in I, \forall k \in K \\
& \left(z_{j_{1} j_{2}}, x_{j_{1}}, x_{j_{2}}\right) \in \mathcal{Z}, \forall j_{1}, j_{2} \in I
\end{aligned}
$$

where $v_{i k}$ and $z_{j_{1} j_{2}}$ are auxiliary decision variables, and $\mathcal{Z}$ and $\mathcal{X}_{i k}$ are feasible regions characterized by linear constraints provided in Appendix B.2, Equations (38) and (39).

Proof of Proposition 1. Please see proof in Appendix B.2. 


\subsection{Operational Profit Model}

One of the key issues in one-way car sharing operations is to maintain service level. The service level $\alpha$ is defined for each region as the probability that customers will find available EVs at their origins when they intend to travel. While we set the same service level $\alpha$ across regions for the ease of discussion, location-specific service level can be easily modeled by introducing $\alpha_{i}$ for region $i$. To maintain the service level, the firm frequently employs "street teams" to reposition vehicles to ensure availability throughout the service region (New York Magazine 2015).

Similar to the rail equipment repositioning model in Adelman (2007), we model fleet operations as a closed queueing network in Figure 3(a). A key feature to note in our modeling is that the EVs, rather than the customers, are the entities that flow within the queueing network, unlike the case in typical models of call centers. In a closed network, the total number of EVs is a constant. At any given time instant, an EV can be in one of four states: (i) being idle at some area awaiting the next customer rental, (ii) traveling from one idle node to another with a customer (i.e., in rental), (iii) traveling from one idle node to another due to repositioning, or (iv) recharging at a charging station. Correspondingly, we define four types of queues to characterize the time delays EVs undergo in these four types of activities. In the following discussion, we use the terms "queue" and "node" interchangeably, as both refer to individual queues in the queueing network.

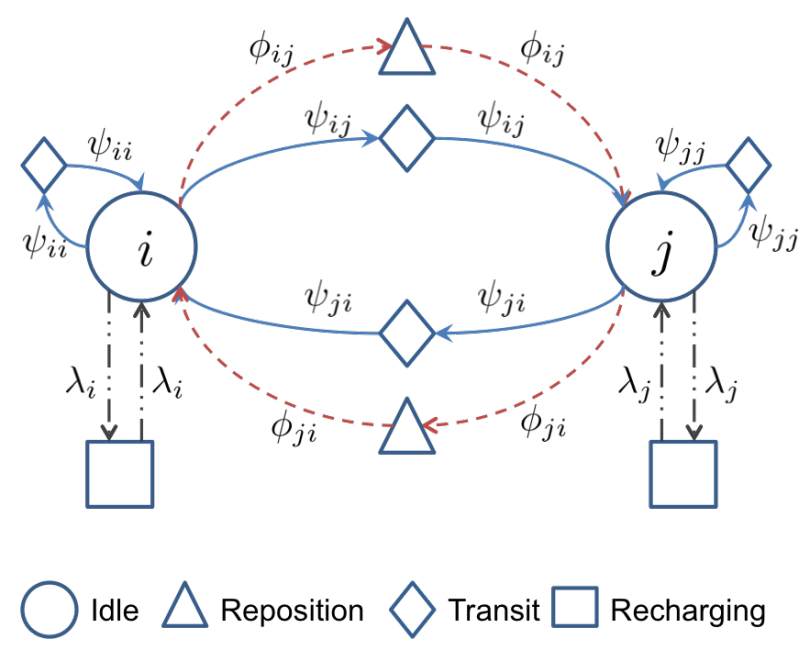

(a) Closed Queueing Network

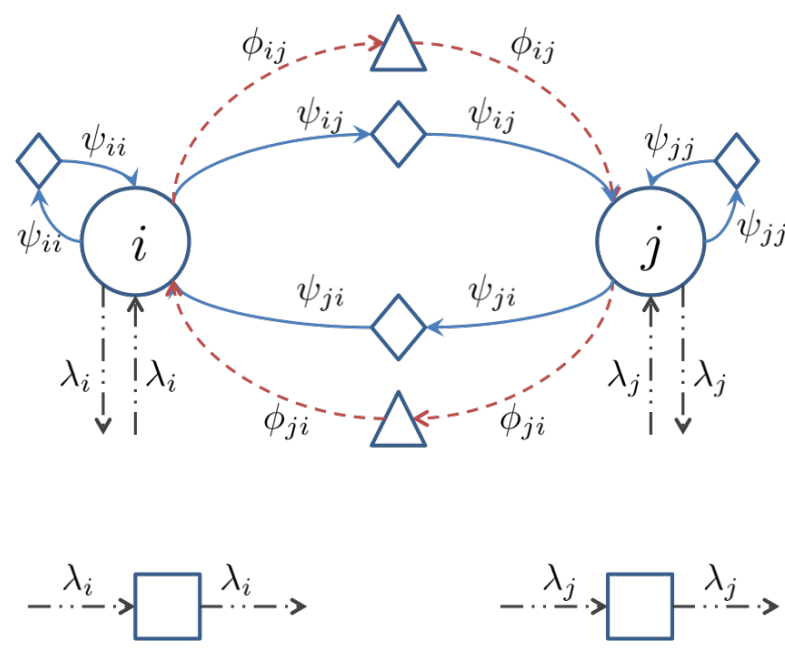

(b) Open Queueing Network

Figure 3 EV Sharing Operations as Queueing Networks 
For ease of exposition, we first discuss the dynamics of the system as if EVs do not need to be repositioned or recharged. After we describe the basic queueing dynamics corresponding to activities (i) and (ii), we will further discuss how activities (iii) and (iv) can be incorporated into the queueuing system.

First, we model each idle node $i$ in the service region as a queue in which EVs in state (i) discussed above remain idle until rented by a customer (Figure 3(a)). We assume that customer requests for trips occur following Poisson processes with origin- and destinationspecific rates. Thus, whenever there is at least one EV in the idle queue, the time until the next rental service starts is exponentially distributed. Assuming that EVs are checked out in a first-in-first-out manner, the total amount time that an EV spends in an idle queue consists of two components: the waiting time until all EVs ahead of it in the queue are checked out (equal to the sum of $m$ independent exponentially-distributed random variables if there are $m$ other EVs ahead), plus the exponentially-distributed waiting time for the rental to begin once reaching the first position in the queue. Because the number of EVs in the queue, as long as it is non-zero, does not affect the rate of departure, the dynamics of EV movements are analogous to a $\cdot / M / 1$ queue in which EVs queue to enter "service" (i.e., wait to reach the first position to be checked out by a customer). Note also that the first-in-first-out assumption is not restrictive, as the EVs are all identical and interchangeable, and we are only interested in the probability that there are no EVs in the queue (and not, for example, the through time of an individual EV). When there are no EVs in the queue, any customer requests (which still follow the same Poisson process) will be lost. The service level, defined as the long-run proportion of customer requests that are met at the idle queue, is thus equal to the probability that the queue is not empty.

When an EV departs from an idle queue $i$ toward the customer's chosen destination $j$, it enters a transit node $(i-j)$, where it remains for a duration (of the customer rental which may depend on the distance between $i$ and $j$ ) following a general distribution. Thus, the transit node is a $\cdot / G / \infty$ queue, in which capacity is considered infinite, as rental durations are independent of one another. After the rental duration, the EV departs the transit node and enters the idle queue $j$.

Note that under the dynamics discussed thus far, the flows of EVs among idle nodes are not necessarily balanced. Thus, repositioning activities (iii) are required to maintain availability of EVs at all locations. To model this, we consider that the EV in the first 
position of an idle queue $i$ can be repositioned to idle queue $j$ following another Poisson process with a rate to be determined by the firm. To capture this, the "service" in the idle queue is now interpreted as waiting for either the next customer rental or the next repositioning trip, whichever occurs first. Because the waiting times (for the first EV in line) for both follow independent exponential distributions, the "service" times at idle queues remain exponentially distributed. If an EV enters a repositioning trip, it travels from $i$ to $j$ while incurring some generally distributed travel time. Thus, the repositioning nodes are also modeled as $\cdot / G / \infty$ queues, with different (faster, as repositioning is to be conducted economically) "service" rates than the corresponding transit queues.

Furthermore, when the battery level of an EV falls below a certain threshold, the EV must be recharged. For tractability, we do not keep track of the battery level for individual EVs. Instead, we assume that every time an EV departs a transit queue (completing a rental), it is re-routed to a recharging queue with a certain probability $P_{c}$ (an exogenous parameter). For ease of discussion, the recharging queue is modeled as an $\cdot / G / \infty$ queue, assuming sufficient charging capacity and generally distributed charging time. Our model can also be easily extended to the case in which there are a finite number of parallel chargers at the recharging queue. Additionally, the travel time between an idle queue and a recharging queue is omitted in the computation, as the fixed-cost infrastructure investment incurred in covering a service area ensures sufficient accessibility of chargers in every service area covered.

Having described the dynamics of the queuing network, we now proceed to model the operational profit function $\Theta(\cdot)$ mathematically. The first step is to characterize the rates of the three classes of EV flows among nodes of the queueing network: transit flows, repositioning flows and recharging flows. Consider the idle queue $i$. Let $\mu_{i}$ (an exogenous parameter) be the maximum outbound trip demand rate, i.e., the demand rate when the adoption rate is equal to one, and let $P_{i j}$ (an exogenous parameter) be the proportion of trips with destination $j$ when all areas are served, where $\sum_{j \in I} P_{i j}=1$. With customer adoption $q_{i k}$ (a decision variable) realized, the trip rate from idle queue $i$ to $j, \Psi_{i j}$ (an auxiliary decision variable) can be written as:

$$
\Psi_{i j}=P_{i j} \mu_{i} \sum_{k \in K} w_{i k} q_{i k}, \forall i, j \in I
$$

where $w_{i k}$ is the weight of customer group $k$ in trip requests. 
Such demand for trips, however, is not necessarily fully satisfied for two reasons. First, those trips with destinations not covered within the service region will not be realized. Second, because the availability of EVs is stochastic, a certain proportion of demand (one minus the service level) will be lost. Let $\psi_{i j}$ (an auxiliary decision variable) be the realized transit flow after accounting for these two factors. Under the service level guarantee, the realized transit flow must be between $\alpha$ (an exogenous parameter) and one times $\Psi_{i j}$, when the destination $j$ is covered in the service region. Therefore, the following constraint holds:

$$
\alpha \Psi_{i j} x_{j} \leq \psi_{i j} \leq \Psi_{i j} x_{j}, \forall i, j \in I .
$$

Furthermore, in the long-run, the same service levels are seen by customers from the same origin, regardless of their destinations. Thus, the proportion of realized transit trips for each destination must equal the proportion of original trip demand for the same destination, as stipulated by the following proportionality constraints (which also appear in the bicycle sharing model of (Shu et al. 2013)):

$$
\psi_{i j}=\frac{P_{i j} x_{j}}{\sum_{l \in I} P_{i l} x_{l}} \sum_{l \in I} \psi_{i l}, \forall i, j \in I .
$$

In (11), note that the fraction $\frac{P_{i j}}{\sum_{l \in I} P_{i l} x_{l}}$ is the proportion of original trip demand to destination $j$ among the destinations covered in the service region, e.g. $x_{j}=1$. We also note that constraints (10) and (11) are nonlinear. To linearize them, we introduce a further set of auxiliary decision variables $\psi_{i j}^{\prime}$ and require them to satisfy the proportionality property $\frac{\psi_{i j}^{\prime}}{\psi_{i l}^{\prime}}=\frac{P_{i j}}{P_{i l}}$ by imposing constraint (14) below. Intuitively, $\psi_{i j}^{\prime}$ gives the potential realized transit trip rate assuming all destinations are covered. With the new set of auxiliary variables, we can replace (10) and (11) with:

$$
\begin{aligned}
& \psi_{i j}^{\prime} \geq \alpha \Psi_{i j}, \forall i, j \in I \\
& \psi_{i j}^{\prime} \leq \Psi_{i j}, \forall i, j \in I \\
& \psi_{i j}^{\prime}=P_{i j} \sum_{l \in I} \psi_{i l}^{\prime}, \forall i, j \in I \\
& \psi_{i j}=\psi_{i j}^{\prime} x_{j}, \forall i, j \in I
\end{aligned}
$$

The nonlinear constraints (15) can further be linearized as the following:

$$
\begin{aligned}
& \psi_{i j} \leq \psi_{i j}^{\prime}, \forall i, j \in I \\
& \psi_{i j} \leq P_{i j} \mu_{i} x_{j}, \forall i, j \in I \\
& \psi_{i j}^{\prime}+P_{i j} \mu_{i}\left(x_{j}-1\right) \leq \psi_{i j}, \forall i, j \in I
\end{aligned}
$$


Recall that an EV is sent to recharge with probability $P_{c}$ when it completes a rental. Thus, the flow rate of an EV to the recharging queue associated with idle queue $i, \lambda_{i}$ (an auxiliary decision variable) is given in the following constraint:

$$
\lambda_{i}=P_{c} \sum_{j \in I} \psi_{j i}, \forall i \in I
$$

Because the realized transit trip rates are not necessarily balanced, repositioning trips are required to maintain availability. Recall that we assume that repositioning trips follow Poisson processes. In particular, we define the decision variable $\phi_{i j}$ as the rate of repositioning trips from $i$ to $j$. Then, the flows of EVs in and out of each idle queue $i$ must satisfy the following flow balance constraint:

$$
\begin{aligned}
\sum_{j \in I} \psi_{i j}+\sum_{j \in I} \phi_{i j} & =\left(1-P_{c}\right) \sum_{j \in I} \psi_{j i}+\sum_{j \in I} \phi_{j i}+\lambda_{i} \\
& =\sum_{j \in I} \psi_{j i}+\sum_{j \in I} \phi_{j i}, \forall i \in I
\end{aligned}
$$

After relating the rates of different types of flows, the next step is to obtain the fleet size required to ensure the guaranteed service level. Recall that at any time instant, an EV can be at any of the four types of nodes (idle, transit, repositioning, recharging) and that the total fleet size thus equals the sum of EVs being held at all these nodes in the closed queueing network. Therefore, relating the rates of flow through each node, which we have just modeled previously, with the number of EVs being held at the same node would allow us to formulate the fleet size requirement. Unfortunately, for a closed queueing network, the relationship between population (fleet) size and flow rates is not straightforward, due to interdependence among flows of different nodes. We apply the fixed population mean (FPM) approximation introduced in Whitt $(1984,2002)$ to circumvent this difficulty. In particular, we approximate the EV population in the closed queueing network by the steady state expected population in a closely-related open queueing network. The open network used for this approximation is illustrated in Figure 3(b). In particular, the idle queues work as M/M/1 queues, and the transit, repositioning and recharging queues work as $\mathrm{M} / \mathrm{G} / \infty$ queues. The network is open because the recharging queues are disconnected from the rest of the network. Thus, at every idle queue $i$, instead of requiring EVs to move to the corresponding recharging queue with rate $\lambda_{i}$, we require that $\mathrm{EVs}$ leave the system 
at the same rate. Correspondingly, at the standalone recharging queue, we assume that external arrivals occur at rate $\lambda_{i}$. Intuitively, this approximation corresponds to relaxing the sample path dependence between the recharging queues with the rest of the system to the case of dependence in expectation.

The open network approximation allows us to relate the fleet size to the flow rate for each node. In particular, with service level $\alpha$, the expected number of EVs awaiting in idle queue $i$ (the queue length) is $\frac{\alpha}{1-\alpha}$. Let $t_{i j}$ and $\tau_{i j}$ (exogenous parameters) be the expected trip durations from $i$ to $j$ for customer rental and repositioning trips, respectively, and $t_{c}$ be the expected time to recharge an EV. By applying Little's law for each queue, the expected fleet size also constitutes the expected values of $\sum_{j \in I} \sum_{i \in I} t_{i j} \psi_{i j} \mathrm{EVs}$ in transit nodes, $\sum_{i \in I} \sum_{j \in I} \tau_{i j} \phi_{i j}$ EVs in repositioning nodes, and $\sum_{i \in I} t_{c} \lambda_{i}$ EVs in recharging queues. Thus, the fleet size $N$ must be no less than the following sum:

$$
\sum_{i \in I} \frac{\alpha}{1-\alpha} x_{i}+\sum_{j \in I} \sum_{i \in I} t_{i j} \psi_{i j}+\sum_{i \in I} \sum_{j \in I} \tau_{i j} \phi_{i j}+\sum_{i \in I} t_{c} \lambda_{i} \leq N
$$

Finally, the last step in formulating our fleet operations submodel is to characterize the operational profit $\Theta(\mathbf{q}, \mathbf{x})$, which consists of four parts: operational revenue, repositioning cost, charging cost, and fleet investment. To ensure consistency in cost accounting, the scaling factor $\xi$ (an exogenous parameter) is applied to unify the time unit, i.e., $\xi=365$ to convert daily rates into yearly rates. The annual operational revenue $\xi \sum_{j \in I} \sum_{i \in I} r t_{i j} \psi_{i j}$ is obtained from EV usage for customer trips between all origin-destination pairs, where $r$ (an exogenous parameter) is the per unit time usage price of an EV. Similarly, the annual repositioning cost is given by $\xi \sum_{i \in I} \sum_{j \in I} \eta \tau_{i j} \phi_{i j}$, where $\eta$ (an exogenous parameter) is the repositioning cost per unit time. Using $c$ (an exogenous parameter) to denote the average cost to fully recharge an $\mathrm{EV}$, the total charging cost is given by $\xi \sum_{i \in I} c \lambda_{i}$. Lastly, we use $h$ (an exogenous parameter) to denote the annually amortized EV purchase cost, which is based on the price and typical life span in the EV sharing fleet. Therefore, the annual operational profit is formulated as:

$$
\Theta(\mathbf{q}, \mathbf{x})=\xi\left(\sum_{j \in I} \sum_{i \in I} r t_{i j} \psi_{i j}-\sum_{i \in I} \sum_{j \in I} \eta \tau_{i j} \phi_{i j}-\sum_{i \in I} c \lambda_{i}\right)-h N
$$


Combining the adoption rate and operational profit submodels, the service region design problem is formulated as a mixed integer second-order cone program (MISOCP):

$$
\max _{\substack{x_{i}, q_{i k}, N, \Psi_{i j} \\ \psi_{i j}, \psi_{i j}^{\prime}, \phi_{i j}, \lambda_{i}}} \sum_{i \in I} \sum_{k \in K} f Q_{i k} q_{i k}-\sum_{i \in I} g_{i} x_{i}+\xi\left(\sum_{j \in I} \sum_{i \in I} r t_{i j} \psi_{i j}-\sum_{i \in I} \sum_{j \in I} \eta \tau_{i j} \phi_{i j}-\sum_{i \in I} c \lambda_{i}\right)-h N
$$

s.t.

$$
\begin{aligned}
& \text { Constraint }(3),(5)-(9),(12)-(14),(16)-(21) \\
& q_{i k}, N, \Psi_{i j}, \psi_{i j}, \psi_{i j}^{\prime}, \phi_{i j}, \lambda_{i} \geq 0 \\
& x_{i} \in\{0,1\}
\end{aligned}
$$

The detailed MISOCP formulation to (22) is shown in Appendix B.3. The above formulation is readily solvable by optimization solvers, such as CPLEX, that can handle MISOCPs.

In practice, the customer travel patterns, including both the trip distribution $P_{i j}$ and outbound trip demand rates $\mu_{i}$, can be time-varying. The proposed model (22) can be extended by incorporating the pointwise stationary approximation (Green and Kolesar 1991) approach as if the travel patterns are stationary at each point in time. The resulting formulation in the presence of time-varying travel patterns is provided in Appendix B.4.

One further point that warrants discussion is the integration of the adoption rate and operational profit submodels. Following Proposition 1, the worst-case adoption rate can be represented by the $q_{i k}$ variables, subject to a set of linear and second-order conic constraints (5)-(7), in the absence of other constraints. However, when the constraints characterizing the queueing network dynamics (which involve the $q_{i k}$ variables) are added, there is no theoretical guarantee that (5) is tight at the optimal solution. While, in practice, we note that the adoption rate constraints (5) in the computational experiments presented in Sec 4 are tight, we also provide Proposition 2 below, which gives sufficient conditions for the tightness of (5).

Proposition 2. For a given service region design $I^{\prime} \subseteq I$, the second-order conic constraint (5) is tight for customer group $k$ in candidate region $i \in I^{\prime}$ when the following sufficient condition holds:

$$
f Q_{i k}+\mu_{i} w_{i k} P_{i i} u_{i i}+\mu_{i} w_{i k} \sum_{\substack{j \in I^{\prime} \\ j \neq i}} P_{i j}\left(u_{i j}-v_{j i}\right)>0
$$


where $u_{i j}=(\xi r-h) t_{i j}-P_{c}\left(\xi c+h t_{c}\right)$ is the expected usage revenue net of the fleet cost and charging cost, and $v_{j i}=(\xi \eta+h) \tau_{j i}$ is the expected reposition cost. In other words, when the expected profit from serving customer group $k$ in candidate region $i$ is positive, the adoption rate constraint is tight.

Proof of Proposition 2. Please see the proof in Appendix B.5.

\section{Case Study: Car2Go in San Diego}

We demonstrate the service region design optimization framework with a case study of Car2Go in San Diego, the first city in North America in which Car2Go is operating an all-EV fleet under the free-floating model. At the time of launch or major re-design, the firm may have access to only limited operations data, such as from pilot studies (from other cities or the current service region) or market surveys, to estimate customer preferences and travel patterns. Our study simulates the case of a service region re-design, in which partial information on travel patterns is available for locations covered in the incumbent service region but not for places not covered currently. We begin by describing the sources of data and the procedures for estimating the parameters for our optimization model.

\subsection{Parameter Estimation}

We consider the set of potential locations to cover, $I$, as the set of 61 zip codes in San Diego county. The values for cost and revenue parameters are taken from Car2Go's website. Those fixed costs and revenue terms (e.g., the one-time membership fee) are amortized to annual costs, assuming a 5-year planning horizon. In particular, the firm earns an annual membership fee of $f=\$ 8$ per customer and a trip revenue of $r=\$ 0.16 / \mathrm{min}$ of usage (adjusted for variable costs). Based on the technical specifications of the Smart Electric Drive, the EV model in the Car2Go fleet, the cost to recharge from a $20 \%$ battery level to full is assumed to be $c=\$ 3$, with a charging time of 6 hours. The probability that an EV needs recharging after a trip is set to $P_{c}=0.2$. In the case of imbalanced flows, the street team must reposition the EVs at a cost of $\$ 0.32 / \mathrm{min}$ (of the duration of the repositioning trip), which corresponds to twice the minimum wage in San Diego. The total repositioning cost depends on the repositioning frequency and distance completed (thus, the duration). Moreover, in our experiments, we assume that an $\alpha=80 \%$ service level is guaranteed throughout the service region.

To estimate the other parameters, particularly those related to travel patterns and fixed costs of covering different candidate locations, we use the following data sets. 
1. Car2Go San Diego operations data. This data set is obtained through web crawling of Car2Go's website between March and April, 2014, and it contains a one-month time stamp record of all idle EVs in the current service region of Car2Go in San Diego at fiveminute intervals. The record includes time, location, battery level and charging status of every idle (available) car. By preprocessing the data, we identify 25,875 trips in total with the current fleet size of 379 EVs. We also verify the Markovian assumption on customer arrivals using the data set and testing procedures in Kim and Whitt (2014).

2. San Diego geographic information and census data. The travel distances and times between all origin-destination pairs are computed using ArcGIS, a geographic information system, with the road network map from the SanGIS data warehouse (SANDAG 2014). The census data are from the 2010 American Community Survey (U.S. Census Bureau 2010), which provides information regarding the working population as well as the per capita income for every zip code in San Diego County.

3. 2010 California Household Travel Survey (CHTS). The CHTS collects travel information from sampled households in all 58 of California's counties (California Department of Transportation 2010). All participating households were first asked to record their travel in a diary for a pre-assigned 24-hour period. For our purposes, we focus on households in San Diego County. We use data from tables associated with households, persons and places, which give the values of relevant attributes, including age, income level, zip codes and modes of trips. In the sample of 1,999 working-age individuals in San Diego County, we identify 6,562 trips, out of which 5,335 were by car.

4. EV charging station information. We use the EV charging station data from (U.S. Department of Energy 2014a), which report attributes such as location, zip code, charger number and EV network for every publicly available EV charging station. We focus on the charging stations in San Diego County that are managed by the EV charging network Blink, which provides charging services to Car2Go.

It is a standard approach in the transportation literature (e.g., Ortúzar and Willumsen (2011, Ch5)) to model trip distribution using gravity models. Using the Car2Go operations data, we fit gravity models for the travel patterns $P_{i j}$ and trip demand rates $\mu_{i}$ for all the candidate regions using the per capita income and working-age population of the respective origins and destinations as independent variables. For the utility parameters $\mathbf{a}_{i}$, we consider their means $\overline{\mathbf{a}}_{i}$ and covariance matrices $\Gamma_{i}$ (which are required for the 
distributionally robust model) to be given by the mean and covariances of $P_{i j}$ obtained from the gravity model regression. Furthermore, to determine the aspirational level $b$, we perform a clustering analysis and identify three groups of travelers based on modal choice in the CHTS data. Excluding the two groups of travelers who mostly travel with their own cars exclusively and those who use public transportation exclusively, we focus on the intermediate group as the likely adopters of car sharing. We consider the aspirational level to be the proportion of trips these travelers complete using their own cars (that can potentially be replaced by car sharing), i.e., $b=0.53$. Details of all estimation procedures are provided in Appendix C.

\subsection{Optimal Service Region}

Using parameter values obtained from the aforementioned sources, we solve the MISOCP in (22) by using CPLEX 12.4 on a 64-bit Windows Operating System equipped with an Intel Core i7 CPU at 2.8GHz and 12GB RAM to obtain the optimal service region and fleet size for the San Diego county candidate service area. The existing service region of Car2Go and the optimal service region obtained from solving the MISOCP are mapped in Figure 4 (a) and (b), respectively. Both solutions agree in covering the downtown area of San Diego, where currently $49.88 \%$ of the recorded trips occurred in Car2Go operations data. The major discrepancy comes between choosing to cover the northern or southern part of the county. The current service region contains Chula Vista city in the south. However, based on the Car2Go operations data, this area only accounted for $1.12 \%$ of all trips. The MISOCP solution suggests covering the northern part of the county, an area with a relatively higher population density and per-capita income, both of which are positively correlated with demand, as suggested by the gravity model. Furthermore, the optimal service region in Figure 4(b) suggests an expansion to cover 35 zip codes (from the existing 18) with a fleet size similar to that in the current system. It is also worth noting that, partially consistent with our model's suggestion, Car2Go recently discontinued service in Chula Vista as of June 30, 2015.

In the following analysis, we compare the environmental benefits of the EV sharing system with the alternative of converting household gasoline cars to EVs. For consistency with previous research, e.g., Lim et al. (2015), we choose to focus on $\mathrm{CO}_{2}$ emissions $\left(\mathrm{CO}_{2} \mathrm{E}\right)$ savings as the measure of environmental benefits. We assume that the EV sharing system operates under the suggested service region in Figure 4(b) 


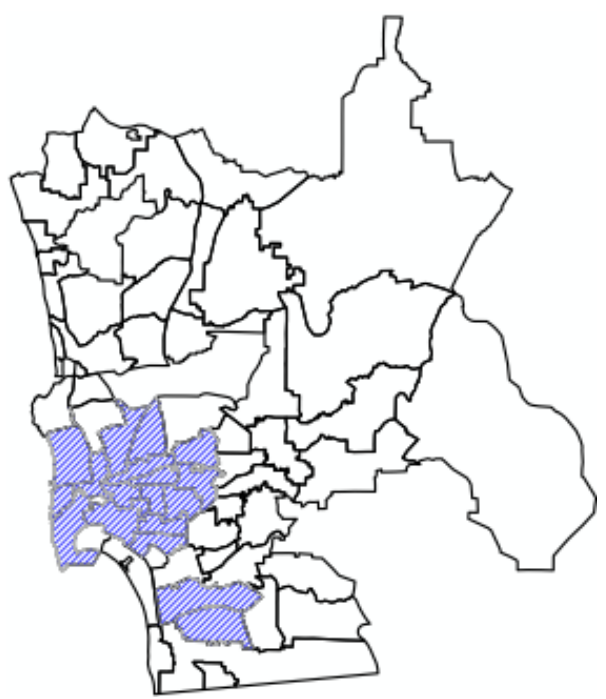

(a) Current Service Region as of Feb, 2015

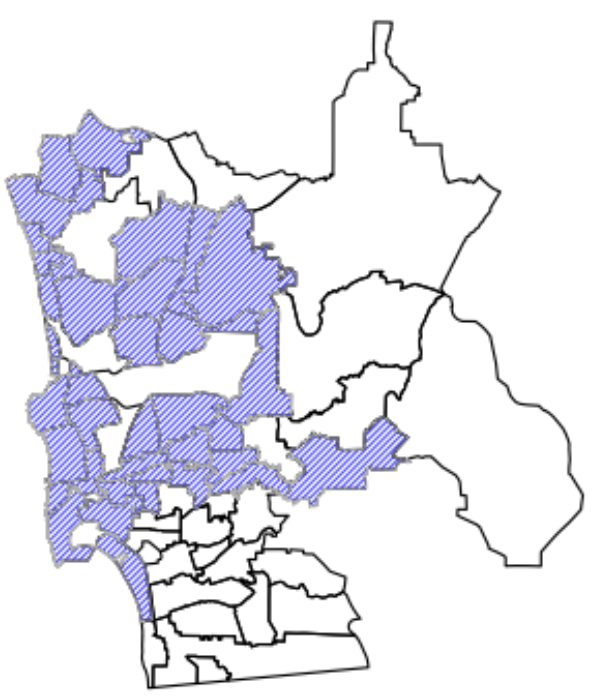

(b) Optimal Service Region

Figure 4 Service Region Designs

with 369 EVs. Considering the adoption rates, opened destinations and service level, the EV sharing system supports $\sum_{i} \sum_{j} \psi_{i j}=957,896.9$ customer trips with a total of $\sum_{i} \sum_{j} \psi_{i j} d_{i j}=13,919,178$ vehicle miles traveled annually, where $d_{i j}$ is the average travel distance from location $i$ to $j$. There are also $\sum_{i} \sum_{j} \phi_{i j}=21,664$ trips with a total of $\sum_{i} \sum_{j} \phi_{i j} d_{i j}=139,879.6$ vehicle miles traveled for fleet repositioning. U.S. Department of Energy (2016) provides the California average well-to-wheel emissions as 0.27 pounds $\mathrm{CO}_{2} \mathrm{E} /$ mile for an $\mathrm{EV}$ and 1.14 pounds $\mathrm{CO}_{2} \mathrm{E} / \mathrm{mile}$ for a gasoline car, assuming an average annual mileage of 10,000 miles. Therefore, the average $\mathrm{CO}_{2}$ emissions savings from an $\mathrm{EV}$ in a sharing fleet is given by $\left[1.14\right.$ (pounds $\left.\mathrm{CO}_{2} \mathrm{E} / \mathrm{mile}\right) \times 13,919,178$ (miles/year) 0.27 (pounds $\mathrm{CO}_{2} \mathrm{E} /$ mile $) \times(13,919,178+139,879.6)($ miles/year $\left.)\right] / 369(\mathrm{EVs})=32,715.22$ pounds $\mathrm{CO}_{2} \mathrm{E} / \mathrm{EV} /$ year. With individually owned cars, replacing a gasoline car with an $\mathrm{EV}$ yields average $\mathrm{CO}_{2}$ emissions savings of $(1.14-0.27)$ (pounds $\left.\mathrm{CO}_{2} \mathrm{E} / \mathrm{mile}\right) \times$ $10,000($ miles $/$ year $)=8,700$ pounds $\mathrm{CO}_{2} \mathrm{E} / \mathrm{EV} /$ year. Therefore, the environmental benefit of an EV in the suggested sharing fleet is $32,715.22 / 8,700=3.76$ times that of an individually owned EV.

OBSERVATION 1. Under the optimal solution in Figure 4(b), each EV in the sharing fleet, on average, yields 3.76 times the $\mathrm{CO}_{2}$ emissions savings of an average individually owned EV. 
Such savings in emissions are mainly due to the higher vehicle utilization in the sharing fleet. Therefore, the amount of savings depends on the geographical characteristics of the service region, customer adoption, trip frequency and travel distances. Our finding echoes the findings of the survey conducted by Firnkorn and Müller (2011), which suggest that there are long-term environmental benefits and vehicle ownership reduction that result from free-floating car sharing systems. Moreover, compared with purchasing EVs, participation in EV sharing systems involves lower opportunity costs and eases consumers' concern over barriers such as range and resale anxieties (Lim et al. (2015)). Thus, EV sharing systems show the potential to deliver GHG emissions savings earlier and to deliver greater cumulative environmental benefits through early adoption.

This finding may also have implications regarding the design of governmental support programs for EVs. In California, car manufacturers are required to sell a certain number of zero emissions vehicles (ZEVs, including EVs) for every conventional car sold or to purchase an equivalent amount of credit from manufacturers who sell a surplus of ZEVs (California Environmental Protection Agency 2014). By selling EVs to its subsidiary Car2Go, Daimler may gain ZEV credits without directly cannibalizing its market for conventional cars (under the Mercedes brand). Observation 1 suggests that such a strategy might help magnify the environmental benefits for the same number of EVs. On the other hand, encouraging travelers (particularly the green-minded population segment) to adopt car sharing might slow down overall EV sales figures and the growth of this nascent segment of production. Thus, it could be an interesting policy question in future studies to evaluate whether the sale of EVs to individuals or to car sharing fleets should be stimulated equally or whether more customized policies should be pursued.

\subsection{Effectiveness of the Robust Solution}

To evaluate the effectiveness of our proposed optimization model (22), we evaluate its performance against three benchmark heuristics. In particular, to assess the value of incorporating customer adoption and fleet operation characteristics in service region optimization, we design the three heuristics based on a more stylized view of the following features:

Assumption 1. The adoption rate at any covered location $q_{s}$ is equal to the aspirational level $b$ and is independent of the selection of other locations. 
Assumption 2. The fleet size required to serve the service region is given by:

$$
N=\bar{t} \times \sum_{i \in I} \mu_{i} q_{i} x_{i}
$$

where $\bar{t}$ is the average travel time per trip and $q_{i}$ is the adoption rate in region $i$.

In Assumption 1, the adoption rate of any covered location is assumed to be equal to the aspirational level because it is estimated as the proportion of trips customers expect to complete with the car sharing service.

We design heuristic $H_{1}$ by making Assumption 1 and utilizing our fleet operations submodel (thus taking a stylized view of customer adoption only); we design $H_{2}$ by making Assumption 2 and utilizing our customer adoption submodel (a stylized view of fleet operations); and we design $H_{3}$ by assuming both (a stylized view of both features). Table 1 summarizes the differences between our model and the heuristics. The heuristic formulations are provided in Appendix D.

Table 1 Benchmarking Heuristics

\begin{tabular}{|c|c|c|}
\hline Heuristic & Treatment & Formulation \\
\hline$H_{1}$ & Adoption rate: replace (5)-(7) with $q_{s}=b$ & MIP \\
\hline$H_{2}$ & Fleet size: replace $(21)$ with $(23)$ & MISOCP \\
\hline$H_{3}$ & Both: replace (5)-(7) and $(21)$ with $q_{s}=b$ and $(23)$ & MIP \\
\hline
\end{tabular}

We generate 20 test instances by randomizing the parameters, $b \in[0.43,0.63]$, the charging power between $[0.8,1.2] \times 3.3 \mathrm{~kW}$ and the service level $\alpha \in[0.75,0.85]$ among 20 instances. For each instance, we solve the service region decisions $\mathbf{x}_{0}, \mathbf{x}_{1}, \mathbf{x}_{2}$ and $\mathbf{x}_{3}$ under our model and the heuristics $H_{1}, H_{2}$ and $H_{3}$, respectively. To provide a fair assessment of solution performances, we evaluate the objective values using simulated adoption rates (using 10,000 samples), assuming that the utility parameters $\mathbf{a}_{i}$ follow an underlying multivariate normal distribution. This assumption ensures a realistic assessment of the performance of distributionally robust models that do not assume knowledge of the true distribution. With simulated adoption rates $\hat{q}_{i k}$ and service region decisions $x_{i}$ previously 
determined, the objective function (profit) for each solution can be evaluated with the following model:

$$
\max _{\substack{N, \Psi_{i j}, \psi_{i j} \\ \psi_{i j}^{\prime}, \phi_{i j}, \lambda_{i} \geq 0}} \sum_{i \in I} \sum_{k \in K} f Q_{i k} \hat{q}_{i k}-\sum_{i \in I} g_{i} x_{i}+\xi\left(\sum_{j \in I} \sum_{i \in I} r t_{i j} \psi_{i j}-\sum_{i \in I} \sum_{j \in I} \eta \tau_{i j} \phi_{i j}-\sum_{i \in I} c \lambda_{i}\right)-h N
$$

s.t.

$$
\text { Constraint (9), (12) - (14), (16) - (21) }
$$

Table 2 summarizes the performance of the approaches under the parameter setting in Sec 4.2 and the randomized instances. Let $\Pi(\mathbf{x})$ be the expected profit from $(24)$ in service region $\mathbf{x}$. We report the percentage gaps between performances of $\mathbf{x}_{0}$ and $\mathbf{x}_{i}$, defined as $\frac{\Pi\left(\mathbf{x}_{0}\right)-\Pi\left(\mathbf{x}_{i}\right)}{\Pi\left(\mathbf{x}_{0}\right)}$.

Table 2 Benchmarking Performance

\begin{tabular}{|c|c|c|c|c|c|c|}
\hline \multirow{2}{*}{ Solution } & \multicolumn{3}{|c|}{ Parameter Setting in Sec 4.2} & \multicolumn{3}{c|}{ Randomized Instances } \\
\cline { 2 - 7 } & Regions Selected & Profit $\left(\times 10^{6} \$\right)$ & Gap & Avg. Gap & Min Gap & Max Gap \\
\hline Our model & 35 & 1.917 & - & - & - & - \\
\hline$H_{1}$ & 21 & 1.576 & $17.81 \%$ & $22.19 \%$ & $12.90 \%$ & $30.56 \%$ \\
\hline$H_{2}$ & 56 & 1.647 & $14.09 \%$ & $18.14 \%$ & $7.78 \%$ & $40.38 \%$ \\
\hline$H_{3}$ & 54 & 1.709 & $10.84 \%$ & $14.78 \%$ & $6.12 \%$ & $37.23 \%$ \\
\hline
\end{tabular}

Under the parameter setting in Sec 4.2, $H_{1}$ underestimates the adoption rates by assuming a uniform system-wide adoption rate $q_{s}$. Because this stylized view fails to account for the fact that adoption rates increase in service region coverage, $H_{1}$ tends to suggest covering fewer locations than is optimal under our proposed model. On the other hand, $\mathrm{H}_{2}$ underestimates the fleet size (and cost) required to cover the service region, as it does not consider the fleet in terms of charging and repositioning. Thus, $H_{2}$ tends to suggest covering more regions than our model. Both $H_{1}$ and $H_{2}$ perform substantially worse than our model in terms of simulated profit. Finally, because $H_{3}$ combines the stylized views on adoption rates and fleet operations, which impose counteracting forces on the size of the service region, it is not surprising that its proposed service region agrees better with that of our proposed model. Overall, by capturing both adoption and fleet operation features 
in detail, our proposed solution outperforms all three heuristics. These observations also highlight the importance of capturing the details of both features, as taking a stylized view on either could lead to significantly worse solutions.

\subsection{Implications of Charging Power, Service Level and Aspirational Level Changes}

In this section, we evaluate the effects of several key modeling parameters on the optimal service region. In particular, we investigate the effects of charging power, service level requirements and aspirational levels. These parameters carry important implications for the key problem characteristics of fleet operations (for the former two) and customer adoption (for the latter).

Due to continuous improvement of battery and charging technologies, the practical charging speed for EVs has improved in recent years. Although the Smart Electric Drive mode that Car2Go uses is only equipped with a $3.3 \mathrm{~kW}$ charger (requiring approximately 6 hours to fully recharge the car), newer models such as the BMW i3 (which DriveNow uses), Renault ZoE, and the Nissan Leaf are compatible with faster chargers with power levels ranging from $7 \mathrm{~kW}$ to $50 \mathrm{~kW}$. Because charging speeds are roughly inversely proportional to charging power (e.g., a $50 \mathrm{~kW}$ quick charger could recharge an EV in approximately 30 minutes), utilizing faster chargers directly reduces the recharging down time and improves EV utilization. To investigate the potential gains from deploying faster chargers, we vary the charging power (and thus, charging times) from our benchmark case and plot the resulting profit, number of covered locations, and fleet density (i.e., fleet size per thousand covered population) in Figure 5.

OBSERVATION 2. Enhancements in charging power from the status quo lead to improvements in service coverage (larger service region) and cost efficiency (smaller fleet density), but with diminishing returns as charging power increases.

Not surprisingly, Figure 5 shows that a faster charging technology enables the firm to serve a larger region with lower fleet density. The major reduction in fleet size comes from the downtime of recharging EVs. Although the general pattern shows that profit improves as charging power increases, the service region size becomes stable when the charging power reaches $20 \mathrm{~kW}$ and above. This finding suggests that, whereas the recharging down time is indeed an obstacle against service region expansion in the status quo, other obstacles such as fixed costs and demand imbalance will factor in to impede further expansion even if 


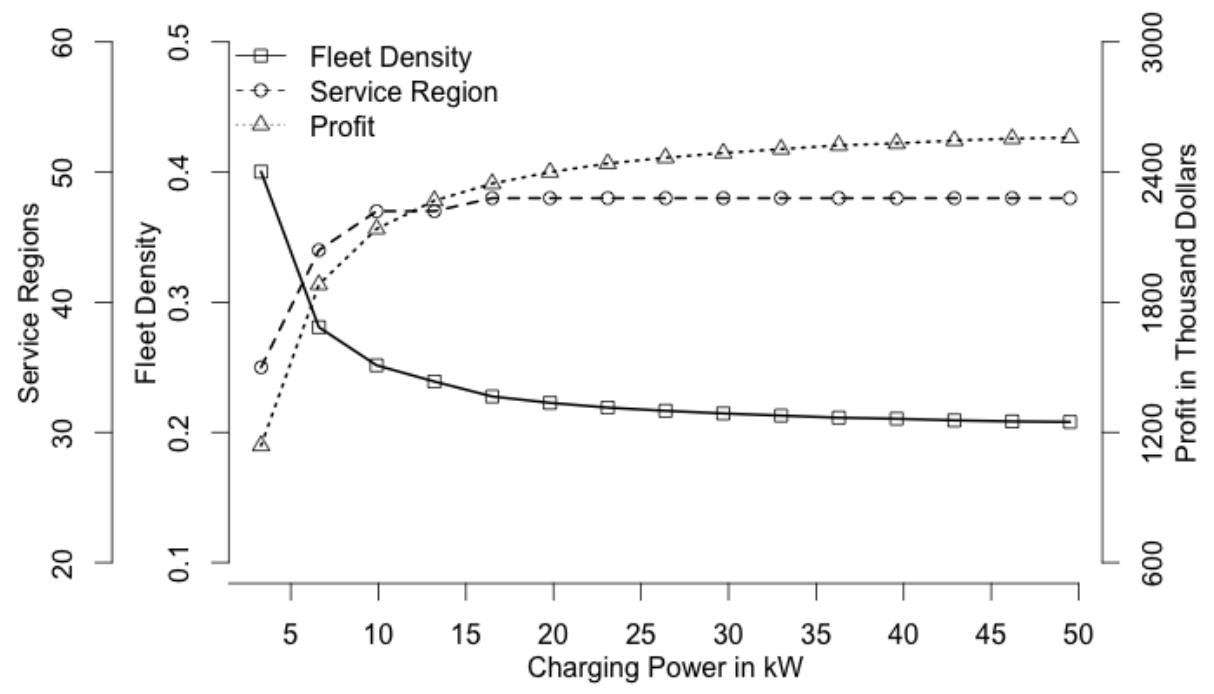

Figure 5 Service Region Design Under Different Charging Power Levels

the charging speed significantly improves. Furthermore, if charging time is interpreted as the total duration of waiting and charging at a charging station, it can further be inferred from Figure 5 that service region coverage and profitability heavily depend upon the development and availability of chargers. When the charging infrastructure is underdeveloped, the total charging and waiting times are long, which might lead to significant shrinkage in service coverage.

Finally, it is also notable that improvements in charging power yield diminishing returns. In practice, high-powered quick charging (e.g., $50 \mathrm{~kW}$ quick charging) is expensive to deploy and may shorten battery life if used regularly. To balance this trade-off, our observation suggests that it is sufficient to deploy medium-speed chargers, such as "Level 2" chargers with 15-20 kW power output, as the potential gains from further improving charging speeds are limited.

Next, we investigate the effects of the service level requirement. Because one primary aim of car sharing services is to replace car ownership, it is of primary concern to ensure high availability of cars such that customers do not encounter significant inconveniences in forgoing (the purchase of) their own cars. On the one hand, a higher service level requirement obviously leads to a larger fleet size and higher costs. On the other hand, it ensures that a higher percentage of demand is satisfied and generates more revenue. In this section, we vary the required service level $\alpha$ and plot the resulting profit, service region size and fleet density in Figure 6. 


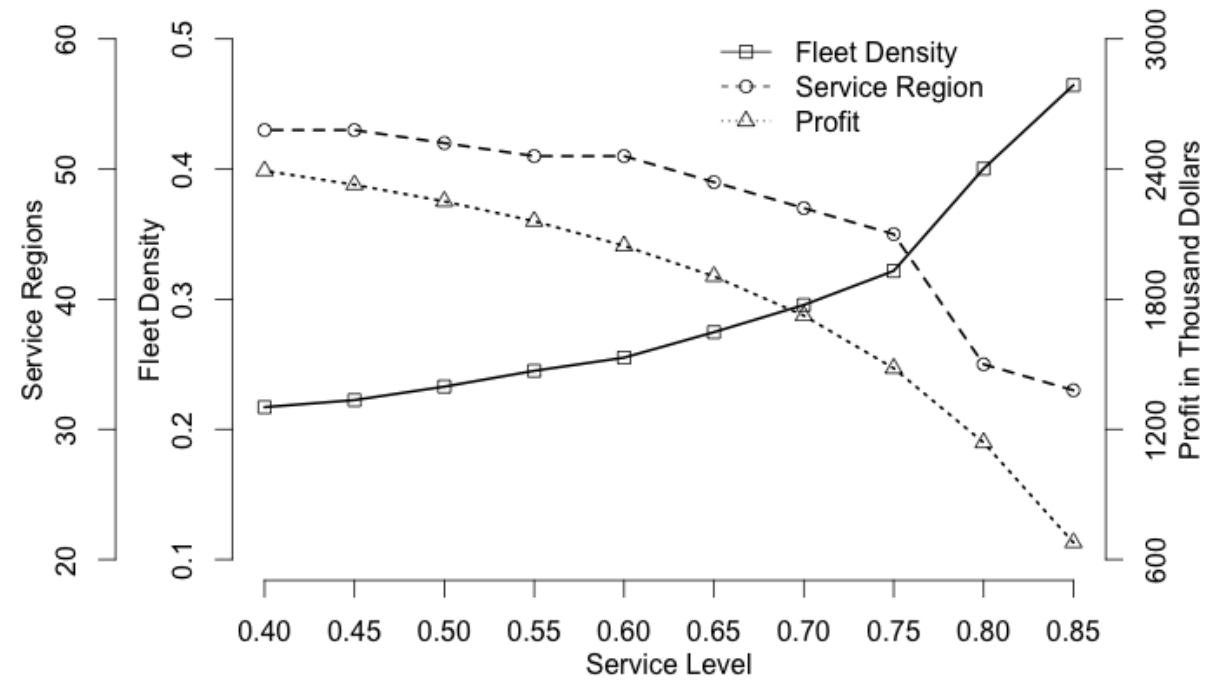

Figure 6 Service Region Design Under Different Service Levels

OBSERVATIOn 3. A higher service level leads to larger fleet investments and a smaller service region with lower profits.

As shown in Figure 6, a higher service level requirement leads to a smaller service region, as the profitability of each service region decreases due to the higher fleet size requirement. Meanwhile, we also note that the profit gains from maintaining a lower service level requirement diminish as $\alpha$ drops to 0.6 or below. Furthermore, in this exercise, we hold the adoption parameter $b$ constant while varying $\alpha$, i.e., we assume that changes in service level do not affect adoption decisions, to better focus on the operational effect of service levels. Nevertheless, our observation indicates that maintaining a low service level only yields marginal improvements in profits. In Section 4.5, we will further evaluate the case in which the adoption threshold, $b$, is dependent on the service level.

Having discussed the impacts of charging power and service level requirements, both of which are major components in the fleet operations submodel, we further test the effects of the aspiration level, $b$, a key parameter in determining customer adoptions. As the aspirational level increases, customers are less likely to adopt the service with the same coverage. It is not immediately clear whether the firm should increase (to encourage more adoptions) or decrease (as marginal revenue of coverage drops) coverage as $b$ increases.

OBSERVATION 4. A higher aspirational level b leads to a larger service region and reduced profit. 


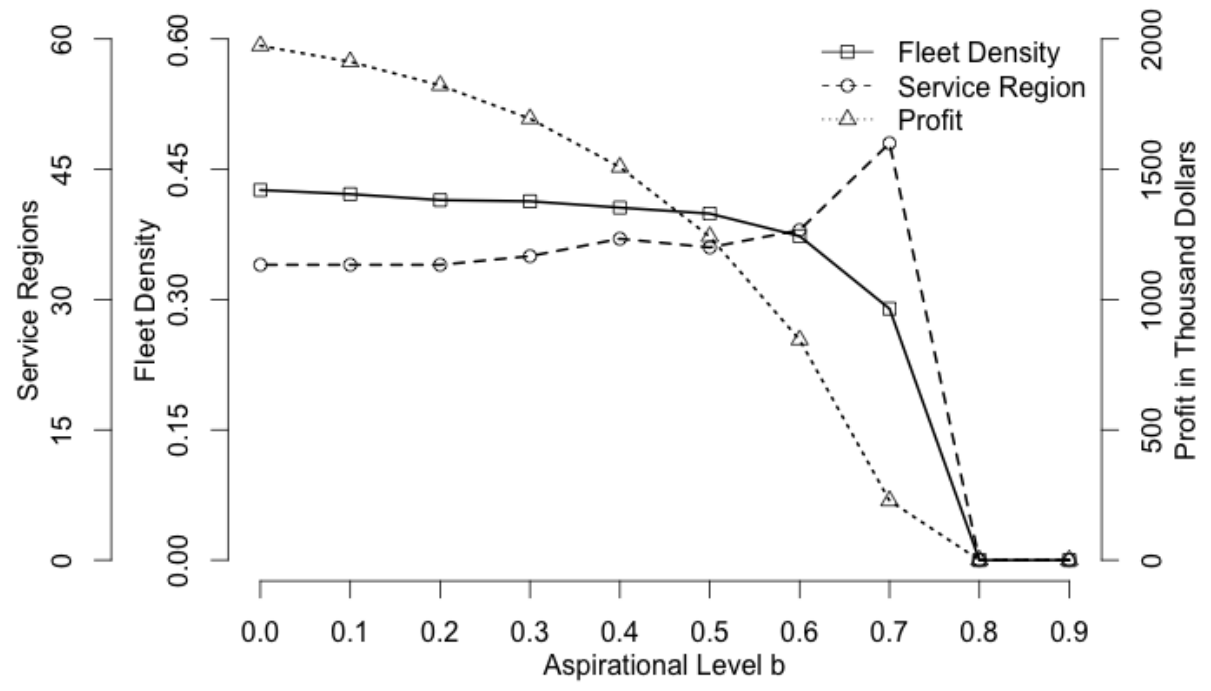

Figure 7 Service Region Design Under Different Aspirational Levels

Figure 7 shows that when the aspirational level is relatively low (e.g., $b \leq 0.5$ ), the service region size is relatively stable, which suggests that in this range, the counteracting forces of expanding the service region to attract demand and shrinking it due to reduced marginal revenue are more or less balanced. Because the aspirational level is medium to moderately high (e.g., $b \leq 0.7$ ), it becomes important to grow the service region to induce adoption as $b$ increases, as the requirement to induce adoption becomes more important. However, as the aspirational level further grows to a very high level (e.g., $b \geq 0.8$ ), customers become too demanding in terms of service coverage, the marginal revenue of coverage becomes too low, and the service thus cannot break even. Therefore, the optimal solution is to not enter the market at all, which also suggests that free-floating car sharing might not be particularly profitable in markets in which customers are completely reliant on driving and expect the car sharing service to fulfill almost all their traveling demands.

\subsection{Price- and Service Level-Sensitive Adoption}

In our baseline optimization model, we model customer adoption behavior as a function of service region coverage (see Section 3.1), assuming that the service level requirement and price level are given constants set at appropriate levels based on market conditions (e.g., a market-competitive price). In practice, there are cases in which these parameters can vary and affect adoption behavior, as a result. In this section, we demonstrate how our optimization model can be applied to tackle this more general case. In particular, to capture the dependence between adoption behavior and these parameters, we allow the aspirational 
level $b$ to be a decreasing function in service level $\alpha$ and an increasing function in price $r$. In this manner, we capture the effects of higher service levels or lower prices attracting higher adoption, holding other parameters constant. In our computational experiments, we assume these functions to be linear, but we note that any general functional form can be easily accommodated using the same approach. In particular, we consider $b=0.53+s_{r}(r-0.16)$ and $b=0.53+s_{\alpha}(0.8-\alpha)$, using the base case parameter values of $b=0.53, r=0.16$ and $\alpha=0.8$ from Sec 4.2 as reference points. For each value of price or service level sensitivity coefficients $\left(s_{r}\right.$ or $\left.s_{\alpha}\right)$, we identify the price and service level that provide the highest expected profits through numerical search. The resulting optimal service regions are plotted in Figures 8(a)-(b).

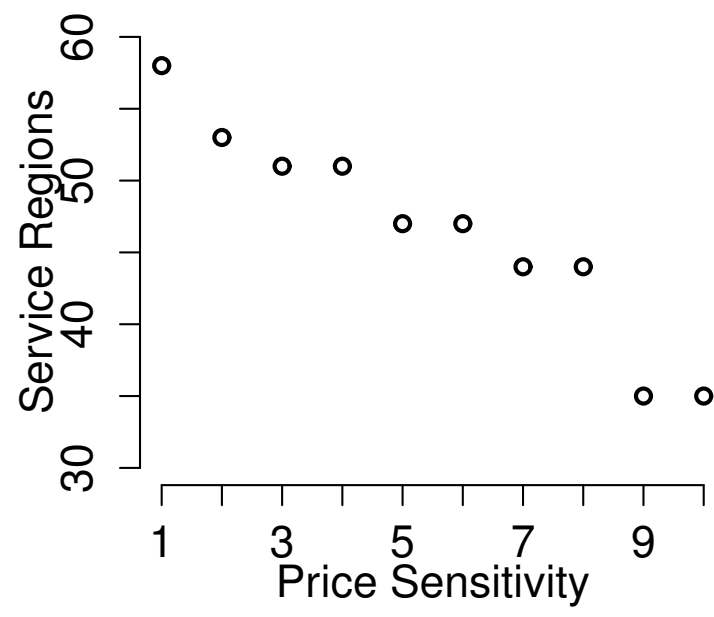

(a) Service Region in Price Sensitivity

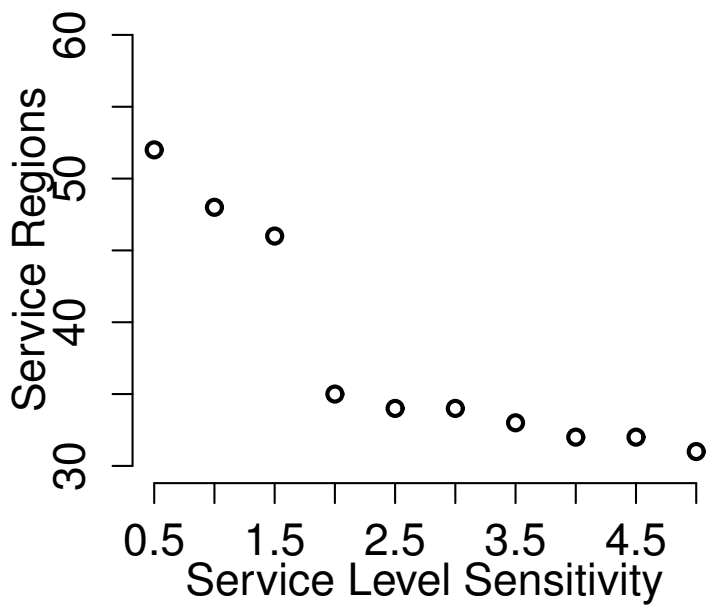

(b) Service Region in Service Level Sensitivity

Figure 8 Price- and Service Level-Sensitive Adoption

Figure 8(a) reports the service region size under optimized prices for price-sensitive adoption. The aspirational level $b$ is set to increase in $r$ at rates $s_{r}$ ranges from 1 to 10 . Figure 8(a) shows that the service region coverage shrinks as the price sensitivity increases. When customers are less price-sensitive, it is profitable to serve a large region to attract more customers and revenue. When the customers are highly price-sensitive, the resulting adoptions are not high enough for the firm to operate profitably in a large region. Therefore, when demand elasticity is high, the price, $r$, should be determined cautiously. 
Figure 8(b) reports the results under service level-sensitive adoption. Because customers are in favor of high service levels, the aspirational level $b$ is decreasing in the service level $\alpha$ at rates $s_{\alpha}$ that range from 0.5 to 5 . When customers are less sensitive to the service level, it is profitable to operate in a large service region with moderate service level target, i.e., attract customers using extensive geographical coverage while offering modest availability to save (fleet) costs. However, as customers' sensitivity to service levels increases, it is optimal to reduce service coverage and provide higher EV availability, and thus the optimal service region tends to shrink. These observations highlight the substitution between attracting customers through the scope (geographical coverage) and quality (availability) of service.

\section{Conclusion}

In this paper, we study the service region design problem for free-floating EV sharing systems. Because customer adoption depends on service coverage at preferred destinations, we explicitly model the adoption decision, which influences the firm's revenue in a probabilistic manner. Under limited information on the utility parameters of destinations, we develop a distributionally robust optimization model to evaluate the adoption rate, aiming to maximize expected profit. We further model the fleet operations, including repositioning and recharging, and determine the fleet size to guarantee the service level in the service region using queueing networks. We provide a lower bound on the expected profit by a computationally tractable MISOCP formulation. Several computational experiments are then conducted to demonstrate the model in a case study of Car2Go San Diego based on real operations data.

Our proposed solutions suggest expansion opportunities under a properly selected service region and optimized fleet size. Because of higher vehicle utilization, EV sharing systems offer more environmental benefits, e.g., savings in $\mathrm{CO}_{2}$ emissions, than replacing personal gasoline cars with EV ownership. The proposed approach also outperforms several heuristics taking stylized views on adoption behavior and fleet size modeling, which suggests the importance of modeling these features in detail. Moreover, our results show that deployment of faster charging equipment can reduce fleet size and expand the service region, albeit with diminishing effects. Our results also suggest that a higher service level leads to a smaller service region to control operating costs, whereas customers with higher aspirational levels lead to a larger service region to induce customer adoption. Finally, we discuss 
how our optimization framework can be applied to identify the optimal price and service levels through numerical experiments in cases in which customer adoption is sensitive to these factors.

While our computational study is set up to simulate a setting in which the firm undergoes a service expansion, we acknowledge that there remain gaps between our study and actual implementation in practice. First, as our trip pattern data are obtained through web crawling, they do not provide information regarding customer characteristics. Thus, we resort to assuming the same trip pattern parameters (means and covariances of $\mathbf{a}_{i}$ ) for all consumer groups residing in the same zip code. Furthermore, we estimate the aspirational level $(b)$ from the modal choice patterns reported for the generic population in the county by the CHTS. In practice, the firm possesses more extensive data on the trip patterns of specific consumers. Through clustering, it will be possible to identify groups of consumers based on their geographical travel patterns and demographic characteristics. Then, the firm may perform targeted surveys with these groups to obtain more accurate, and segment-specific, assessments of their aspirational levels.

In our demonstration of joint price (service levels) and service region optimization in Section 4.5, we note that the sensitivities of adoption with respect to price and service level are not precisely modeled or estimated. In practice, the firm is in possession with more data (e.g., from surveys or focus groups studies) that could be used to obtain better estimates. Even if such data is not available, the firm could engage in small-scale experiments by varying prices and service levels in certain areas and measuring the changes in adoption. Therefore, we believe that our modeling framework has potential to be further enhanced when equipped with more comprehensive data.

When the service region is well planned, the next challenge for car sharing systems is to maintain efficient operations while providing a high-quality customer experience. One particular focus in operations improvement is on the management of car availability and reservations. For instance, Car2Go customers can reserve cars online up to 30 minutes ahead of their trip(Car2Go 2014). It would be interesting to investigate whether such a reservation policy facilitates efficient fleet management. Efficient reservation policies, e.g., allowing reservation terms and possibly fees to depend on time and the availability of cars, may also help balance the customer trips and reduce repositioning efforts without jeopardizing the service level. Therefore, a future research direction will be to explore the effects of reservation policies on EV utilization, availability and profitability. 


\section{References}

ACCESS. 2011. The impact of carsharing on household vehicle ownership. http://www. uctc.net/access/ 38/access38_carsharing_ownership.shtml.

Adelman, Daniel. 2007. Price-directed control of a closed logistics queueing network. Operations Research 55(6) $1022-1038$.

Agrawal, Vishal, Ioannis Bellos. 2016. The potential of servicizing as a green business model. Management Science Forthcoming.

Ang, Marcus, Yun Fong Lim, Melvyn Sim. 2012. Robust storage assignment in unit-load warehouses. Management Science 58(11) 2114-2130.

Atcheson, John, Logan Green. 2012. Car sharing and pooling: Reducing car over-population and collaborative consumption. http://energyseminar.stanford.edu/node/425.

Autolib. 2016. https://www. autolib.eu/en/.

Avci, Buket, Karan Girotra, Serguei Netessine. 2014. Electric vehicles with a battery switching station: Adoption and environmental impact. Management Science .

Banerjee, Siddhartha, Carlos Riquelme, Ramesh Johari. 2015. Pricing in ride-share platforms: A queueingtheoretic approach. Working paper .

Bellos, Ioannis, Mark Ferguson, L Beril Toktay. 2015. To sell and to provide? the economic and environmental implications of the auto manufacturer's involvement in the Car Sharing Business. Working Paper .

Ben-Tal, Aharon, Arkadi Nemirovski. 1998. Robust convex optimization. Mathematics of Operations Research 23(4) 769-805.

Ben-Tal, Aharon, Arkadi Nemirovski. 1999. Robust solutions of uncertain linear programs. Operations research letters $\mathbf{2 5}(1)$ 1-13.

Benjaafar, Saif, Guangwen Crystal Kong, Xiang Li, Costas Courcoubetis. 2015. Peer-to-peer product sharing: Implications for ownership, usage and social welfare in the sharing economy. Working paper .

Bertsimas, Dimitris, Melvyn Sim. 2003. Robust discrete optimization and network flows. Mathematical programming 98(1-3) 49-71.

Bertsimas, Dimitris, Melvyn Sim. 2004. The price of robustness. Operations research 52(1) 35-53.

Boyacı, Burak, Konstantinos G Zografos, Nikolas Geroliminis. 2015. An optimization framework for the development of efficient one-way car-sharing systems. European Journal of Operational Research 240(3) $718-733$.

Bruglieri, Maurizio, Alberto Colorni, Alessandro Luè. 2014. The vehicle relocation problem for the one-way electric vehicle sharing: an application to the milan case. Procedia-Social and Behavioral Sciences 111 $18-27$. 
Burer, Samuel. 2009. On the copositive representation of binary and continuous nonconvex quadratic programs. Mathematical Programming 120(2) 479-495.

Cachon, Gerard P, Kaitlin M Daniels, Ruben Lobel. 2015. The role of surge pricing on a service platform with self-scheduling capacity. Working paper .

California Department of Transportation. 2010. "California Household Travel Survey". http: //www. dot. ca.gov/hq/tsip/otfa/tab/chts_travelsurvey.html.

California Environmental Protection Agency. 2014. "Zero Emission Vehicle (ZEV) Program". http: //www . arb.ca.gov/msprog/zevprog/zevprog.htm.

California Plug-in Electric Vehicle Collaborative. 2016. Charging equipment costs. http://driveclean . ca.gov/pev/Costs/Charging_Equipment.php.

Car2Go. 2014. Car2Go Trip Process Agreement (U.S.). https://www.car2go.com/global-tc/us/ us-en-fa.pdf.

Car2Go. 2015. Car2Go San Diego. https://www.car2go.com/en/sandiego/.

Car2Go. 2016. https://www. car2go.com/.

Center for Climate and Energy Solutions. 2014. U.S. Emissions. http://www.c2es.org/energy/use/ transportation.

Chen, Wenqing, Melvyn Sim, Jie Sun, Chung-Piaw Teo. 2010. From cvar to uncertainty set: Implications in joint chance-constrained optimization. Operations research 58(2) 470-485.

Chen, Xin, Melvyn Sim, Peng Sun. 2007. A robust optimization perspective on stochastic programming. Operations Research 55(6) 1058-1071.

de Almeida Correia, Gonçalo Homem, António Pais Antunes. 2012. Optimization approach to depot location and trip selection in one-way carsharing systems. Transportation Research Part E: Logistics and Transportation Review 48(1) 233-247.

Delage, Erick, Yinyu Ye. 2010. Distributionally robust optimization under moment uncertainty with application to data-driven problems. Operations research 58(3) 595-612.

DriveNow. 2016. https://us.drive-now.com/en/.

El Ghaoui, Laurent, Maksim Oks, Francois Oustry. 2003. Worst-case value-at-risk and robust portfolio optimization: A conic programming approach. Operations Research 51(4) 543-556.
Engadget. 2014. Car2go brings north america's first all-electric carshar- ing program to san diego. http://www.engadget.com/2011/07/14/ car2go-brings-north-americas-first-all-electric-carsharing-prog/.

Febbraro, Angela, Nicola Sacco, Mahnam Saeednia. 2012. One-way carsharing: solving the relocation problem. Transportation Research Record: Journal of the Transportation Research Board (2319) 113-120. 
Firnkorn, Jörg, Martin Müller. 2011. What will be the environmental effects of new free-floating car-sharing systems? the case of car2go in ulm. Ecological Economics 70(8) 1519-1528.

Flath, Christoph M, Jens P Ilg, Sebastian Gottwalt, Hartmut Schmeck, Christof Weinhardt. 2013. Improving electric vehicle charging coordination through area pricing. Transportation Science 48(4) 619-634.

Fraiberger, Samuel P, Arun Sundararajan. 2015. Peer-to-peer rental markets in the sharing economy. NYU Stern School of Business Research Paper .

Goh, Joel, Melvyn Sim. 2010. Distributionally robust optimization and its tractable approximations. Operations Research 58(4-part-1) 902-917.

Green, Linda, Peter Kolesar. 1991. The pointwise stationary approximation for queues with nonstationary arrivals. Management Science 37(1) 84-97.

Hartigan, John A, Manchek A Wong. 1979. Algorithm AS 136: A k-means clustering algorithm. Applied statistics $100-108$.

Hu, Ming, Yun Zhou. 2016. Dynamic type matching. Working Paper .

Jiang, Baojun, Lin Tian. 2016. Collaborative consumption: Strategic and economic implications of product sharing. Management Science. Forthcoming .

John Kleint. 2016. Cityspeed - road network efficiency via online mapping. http://cityspeed. sourceforge.net/.

Kabra, Ashish, Elena Belavina, Karan Girotra. 2015. Bike share systems: Accessibility and availability. Working Paper .

Kim, Song-Hee, Ward Whitt. 2014. Are call center and hospital arrivals well modeled by nonhomogeneous poisson processes? Manufacturing \& Service Operations Management 16(3) 464-480.

Kleindorfer, Paul R, Kalyan Singhal, Luk N Wassenhove. 2005. Sustainable operations management. Production and operations management 14(4) 482-492.

Kong, Qingxia, Chung-Yee Lee, Chung-Piaw Teo, Zhichao Zheng. 2013. Scheduling arrivals to a stochastic service delivery system using copositive cones. Operations Research 61(3) 711-726.

Lim, Michael K, Ho-Yin Mak, Ying Rong. 2015. Toward mass adoption of electric vehicles: Impact of the range and resale anxieties. Manufacturing \& Service Operations Management 17(1) 101-119.

Mak, Ho-Yin, Ying Rong, Zuo-Jun Max Shen. 2013. Infrastructure planning for electric vehicles with battery swapping. Management Science 59(7) 1557-1575.

Mak, Ho-Yin, Ying Rong, Jiawei Zhang. 2014. Appointment scheduling with limited distributional information. Management Science 61(2) 316-334.

Mak, Ho-Yin, Zuo-Jun Max Shen. 2014. Pooling and dependence of demand and yield in multiple-location inventory systems. Manufacturing 83 Service Operations Management 16(2) 263-269. 
Natarajan, Karthik, Melvyn Sim, Joline Uichanco. 2010. Tractable robust expected utility and risk models for portfolio optimization. Mathematical Finance 20(4) 695-731.

Natarajan, Karthik, Chung Piaw Teo, Zhichao Zheng. 2011. Mixed 0-1 linear programs under objective uncertainty: A completely positive representation. Operations research 59(3) 713-728.

New York Magazine. 2015. Can car2go transform new york into a city of drivers? http://nymag.com/ daily/intelligencer/2015/01/car2go-car-sharing-new-york.html.

Nourinejad, Mehdi, Sirui Zhu, Sina Bahrami, Matthew J Roorda. 2015. Vehicle relocation and staff rebalancing in one-way carsharing systems. Transportation Research Part E: Logistics and Transportation Review 81 98-113.

Ortúzar, Juan de Dios, Luis G Willumsen. 2011, Ch5. Modelling transport. John Wiley \& Sons.

Plambeck, Erica L. 2013. Om forum-operations management challenges for some cleantech firms. Manufacturing $\&$ Service Operations Management 15(4) 527-536.

ReachNow. 2016. http://www. bmwcarsharing.com/.

Reuters. 2013. Carsharing services will surpass 12 million members worldwide by 2020, forecasts navigant research. http://www.reuters.com/article/2013/08/22/ co-navigant-research-idUSnBw225991a+100+BSW20130822.

SANDAG. 2014. SanGIS/SANDAG data warehouse". http://www.sangis.org/Download_GIS_Data.htm.

Schneider, Michael, Andreas Stenger, Dominik Goeke. 2014. The electric vehicle-routing problem with time windows and recharging stations. Transportation Science 48(4) 500-520.

See, Chuen-Teck, Melvyn Sim. 2010. Robust approximation to multiperiod inventory management. Operations research $\mathbf{5 8 ( 3 )} 583-594$.

Shu, Jia, Mabel C Chou, Qizhang Liu, Chung-Piaw Teo, I-Lin Wang. 2013. Models for effective deployment and redistribution of bicycles within public bicycle-sharing systems. Operations Research 61(6) 13461359.

Simon, Herbert A. 1957. Models of Man: Social and Rational. Wiley.

Tang, Christopher S, Jiaru Bai, Kut C So, Xiqun Michael Chen, Hai Wang. 2016. Coordinating supply and demand on an on-demand platform: Price, wage, and payout ratio. Working paper .

Taylor, Terry. 2016. On-demand service platforms. Working paper .

The Economist. 2013. The rise of the sharing economy. http://www.economist.com/news/leaders/ 21573104-internet-everything-hire-rise-sharing-economy.

U.S. Census Bureau. 2010. 2010 american community survey". http://www.census .gov/acs.

U.S. Department of Energy. 2014a. Alternative Fuels Data Center. http://www.afdc.energy.gov/data_ download/. 
U.S. Department of Energy. 2014b. Charging Plug-In Electric Vehicles at Home. http: //www . afdc. energy . gov/fuels/electricity_charging_home.html.

U.S. Department of Energy. 2016. Emissions from hybrid and plug-in electric vehicles. http://www. afdc . energy.gov/vehicles/electric_emissions.php.

U.S. Environmental Protection Agency. 2014. Sources of Greenhouse Gas Emissions. http://www.epa.gov/ climatechange/ghgemissions/sources/transportation.html.

Weikl, Simone, Klaus Bogenberger. 2013. Relocation strategies and algorithms for free-floating car sharing systems. Intelligent Transportation Systems Magazine, IEEE 5(4) 100-111.

Whitt, Ward. 1984. Open and closed models for networks of queues. AT\&T Bell Laboratories Technical Journal 63(9) 1911-1979.

Whitt, Ward. 2002. Stochastic-Process Limits: An Introduction to Stochastic-Process Limits and Their Application to Queues. Springer.

Wills, Michael J. 1986. A flexible gravity-opportunities model for trip distribution. Transportation Research Part B: Methodological 20(2) 89-111. 


\section{Online Supplement}

\section{Appendix A: Summary of the Notation}

\section{Table 3 Notation}

\begin{tabular}{|c|c|c|}
\hline Parameter & Units & Definition \\
\hline$f$ & $\$$ & fixed (annual) membership fee \\
\hline$Q_{i k}$ & Scalar & Population of customer group $k$ in area $i$ \\
\hline$g_{i}$ & $\$$ & Fixed cost of serving area $i$ \\
\hline$r$ & $\$$ per unit time per $\mathrm{EV}$ & Usage based price of the service \\
\hline$t_{i j}$ & unit of time & Expected travel time to destination $j$ from origin $i$ \\
\hline$c$ & $\$$ & Average charging cost each time \\
\hline$\eta$ & $\$$ per unit of time & Repositioning cost \\
\hline$\tau_{i j}$ & unit of time & Expected reposition time to destination $j$ from origin $i$ \\
\hline$h$ & $\$$ & Annually amortized EV cost \\
\hline$a_{i j}$ & \multirow{2}{*}{$\begin{array}{c}\text { Scalar random variable } \\
\text { Scalar }\end{array}$} & Utility of serving destination $j$ for a customer in area $i$ \\
\hline $\bar{a}_{i j}$ & & Expected value of $a_{i j}$ \\
\hline$\Gamma_{i}$ & Scalar & Covariance matrix of $a_{i j}$ for area $i$ \\
\hline$b_{k}$ & Scalar & Aspirational level for a customer in group $k$ to adopt the service \\
\hline$\mu_{i}$ & Trips per unit of time & Outbound trip rate from area $i$ \\
\hline$P_{i j}$ & {$[0,1]$} & Probability of trip destination $j$ given the origin $i$ \\
\hline$w_{i k}$ & \multirow{2}{*}{$\begin{array}{l}{[0,1]} \\
(0,1)\end{array}$} & Weight (in trip) of customer group $k$ in area $i$ \\
\hline$\alpha$ & & Service level \\
\hline$P_{c}$ & $(0,1)$ & Probability of an arrival EV needs recharge \\
\hline$t_{c}$ & unit time per EV & Average charging time per EV \\
\hline \multicolumn{2}{|c|}{ Decision Variables } & Definition \\
\hline \multicolumn{2}{|r|}{$x_{i}$} & 1 if area $i$ is served; 0 otherwise. \\
\hline \multicolumn{2}{|r|}{$q_{i k}$} & Adoption rate of customer group $k$ in area $i$ \\
\hline \multicolumn{2}{|r|}{$N$} & Fleet size \\
\hline \multicolumn{2}{|r|}{$\Psi_{i j}$} & Trip demand rate from $i$ to $j$ \\
\hline \multicolumn{2}{|r|}{ Trips pe } & Satisfied trip rate if $j$ is served \\
\hline \multicolumn{2}{|r|}{ Trips pe } & Satisfied trip rate: $\psi_{i j}=\psi_{i j}^{\prime} x_{j}$ \\
\hline \multicolumn{2}{|r|}{ Trips pe } & Repositioning trip rate from $i$ to $j$ \\
\hline \multicolumn{2}{|r|}{ Trips per } & EV arrival rate at charging stations in area $i$ \\
\hline
\end{tabular}

\section{Appendix B: Proofs of Analytical Results}

\section{B.1. Proof of Lemma 1}

We begin the proof with deriving the formulation for the worst-case adoption rate in (4) following a similar approach as in El Ghaoui et al. (2003). For ease of exposition, it is safe to temporarily drop the index $i \in I$ and $k \in K$. Given the service region decision $\mathbf{x}$, solving the following optimization problem yields the worst-case non-adoption rate $V(\mathbf{x})=\sup \operatorname{Prob}\left(\sum_{j \in J} a_{i j} x_{j} \leq b_{k}\right)$ :

$$
\begin{aligned}
& \max \mathbb{E}[I(\mathbf{a})] \\
& \text { s.t. } \\
& \int_{\mathbb{R}_{+}^{n}}\left[\begin{array}{l}
\mathbf{a} \\
1
\end{array}\right]\left[\begin{array}{l}
\mathbf{a} \\
1
\end{array}\right]^{T} p(\mathbf{a}) d \mathbf{a}=\mathbf{\Sigma}
\end{aligned}
$$

where $p$ is the probability density function and $I(\mathbf{a})$ is the indicator function defined as

$$
I(\mathbf{a})=\left\{\begin{array}{lr}
1, & \text { if } \\
0, & \text { otherwise }
\end{array}\right.
$$


We write the Lagrange function with symmetric multiplier matrix $M \in \mathcal{S}_{n+1}$ as:

$$
\begin{aligned}
L(p, \mathbf{M}) & =\int_{\mathbb{R}_{+}^{n}} I(\mathbf{a}) p(\mathbf{a}) d \mathbf{a}+\left\langle\mathbf{M}, \boldsymbol{\Sigma}-\int_{\mathbb{R}_{+}^{n}}\left[\begin{array}{l}
\mathbf{a} \\
1
\end{array}\right]\left[\begin{array}{l}
\mathbf{a} \\
1
\end{array}\right]^{T} p(\mathbf{a}) d \mathbf{a}\right\rangle \\
& =\langle\mathbf{M}, \boldsymbol{\Sigma}\rangle+\int_{\mathbb{R}_{+}^{n}}(I(\mathbf{a})-l(\mathbf{a})) p(\mathbf{a}) d \mathbf{a}
\end{aligned}
$$

where $l(\mathbf{a})=\left[\begin{array}{ll}\mathbf{a} & 1\end{array}\right] \mathbf{M}[\mathbf{a} 1]^{T}$. Since $\boldsymbol{\Sigma} \succ 0$, strong duality holds. Therefore, we have

where

$$
V(\mathbf{x})=\inf _{\mathbf{M}=\mathbf{M}^{T}} \sup _{p} L(p, \mathbf{M})
$$

$$
\begin{aligned}
& \sup _{p} L(p, \mathbf{M})=\langle\mathbf{M}, \boldsymbol{\Sigma}\rangle+\sup _{p \in \mathcal{P}} \int_{\mathbb{R}_{+}^{n}}(I(\mathbf{a})-l(\mathbf{a})) p(\mathbf{a}) d \mathbf{a} \\
& =\left\{\begin{array}{lr}
\langle\mathbf{M}, \boldsymbol{\Sigma}\rangle, & \text { if } I(\mathbf{a})-l(\mathbf{a}) \leq 0, \forall \mathbf{a} \in \mathbb{R}_{+}^{n} \\
+\infty, & \text { otherwise. }
\end{array}\right.
\end{aligned}
$$

$V(\mathbf{x})$ is finite if and only if $I(\mathbf{a})-l(\mathbf{a}) \leq 0, \forall \mathbf{a} \in \mathbb{R}_{+}^{n}$. There are two cases:

1. $l(\mathbf{a}) \geq 0, \forall \mathbf{a} \in \mathbb{R}_{+}^{n}$.

2. $l(\mathbf{a}) \geq 1, \forall \mathbf{a} \in \mathbb{R}_{+}^{n}$ such that $\sum_{j \in J} a_{j} x_{j} \leq b$.

Case 1 is equivalent to $\mathbf{M} \succeq_{c o} 0$ and Case 2 holds if there exist a scalar $\tau \geq 0$ such that, $l(\mathbf{a}) \geq 1-2 \tau\left(\mathbf{a}^{T} \mathbf{x}-b\right)$. In addition, the Slater's condition is satisfied as there exist an a such that $\sum_{j \in J} a_{j} x_{j}<b$. As a result, the above constraint for Case 2 is also sufficient. Equivalently, it can be written as $\mathbf{M}+\left[\begin{array}{cc}0 & \tau \mathbf{x} \\ \tau \mathbf{x}^{T} & -1-2 \tau b\end{array}\right] \succeq_{\text {co }} 0$.

$V(\mathbf{x})$ is then the solution to the following copositive program $(\mathrm{CP})$ :

$$
\begin{aligned}
V(\mathbf{x})= & \min \langle\mathbf{M}, \boldsymbol{\Sigma}\rangle \\
\text { s.t. } & \\
& \tau \geq 0 \\
& \mathbf{M} \succeq_{c o} 0 \\
& \mathbf{M}+\left[\begin{array}{cc}
0 & \tau \mathbf{x} \\
\tau \mathbf{x}^{T} & -1-2 \tau b
\end{array}\right] \succeq_{c o} 0 .
\end{aligned}
$$

We then complete the proof by restoring the indices $i \in I$ and $k \in K$ and replacing $V(\mathbf{x}) \leq 1-q($ as $V(\mathbf{x})$ is the non-adoption rate) with the above $\mathrm{CP}$ :

$$
\begin{aligned}
& \left\langle\mathbf{M}_{i k}, \boldsymbol{\Sigma}_{i}\right\rangle \leq 1-q \\
& \tau_{i k} \geq 0 \\
& \mathbf{M}_{i k} \succeq_{c o} 0 \\
& \mathbf{M}_{i k}+\left[\begin{array}{cc}
0 & \tau_{i k} \mathbf{x} \\
\tau_{i k} \mathbf{x}^{T} & -1-2 \tau_{i k} b_{k}
\end{array}\right] \succeq_{c o} 0
\end{aligned}
$$

The last step is to linearize the term $\tau_{i k} \mathbf{x}$. Since $\mathbf{x}$ is a binary vector and $\tau_{i k}$ is continuous, we can replace the term $\tau_{i k} \mathbf{x}$ by vector $\mathbf{d}_{i k}$ with the following constraints:

$$
\begin{aligned}
& -\rho \mathbf{x} \leq \mathbf{d}_{i k} \\
& \mathbf{d}_{i k} \leq \rho \mathbf{x} \\
& \tau_{i k} \mathbf{e}+\rho(\mathbf{x}-\mathbf{e}) \leq \mathbf{d}_{i k} \\
& \mathbf{d}_{i k} \leq \tau_{i k} \mathbf{e}+\rho(\mathbf{e}-\mathbf{x})
\end{aligned}
$$

where $\rho$ is a large scalar and $\mathbf{e}$ is the vector of ones. This completes the proof. 


\section{B.2. Proof of Proposition 1}

By restricting the copositive constraints with semidefinite constraints, we obtain a lower bound to the optimal solution to the formulation with copositive constraints in Lemma 1 . When $\Theta(\mathbf{q}, \mathbf{x})$ is linear, the following formulation is a mixed integer semidefinite program (MISDP):

$$
\begin{aligned}
\max _{q_{i k}, x_{i}, \mathbf{M}_{i k}, \tau_{i k}, \mathbf{d}_{i k}} & \sum_{i \in I} \sum_{k \in K} f Q_{i k} q_{i k}-\sum_{i \in I} g_{i} x_{i}+\Theta(\mathbf{q}, \mathbf{x}) \\
\text { s.t. } & \\
& \left\langle\mathbf{M}_{i k}, \boldsymbol{\Sigma}_{i k}\right\rangle \leq 1-q_{i k}, \forall i \in I, \forall k \in K \\
& \mathbf{M}_{i k} \succeq 0, \forall i \in I, \forall k \in K \\
& \mathbf{M}_{i k}+\left[\begin{array}{l}
0 \\
\mathbf{d}_{i k}^{T}-1-2 \tau_{i k} b_{k}
\end{array}\right] \succeq 0, \forall i \in I, \forall k \in K \\
& -\rho \mathbf{x} \leq \mathbf{d}_{i k}, \forall i \in I, \forall k \in K \\
& \mathbf{d}_{i k} \leq \rho \mathbf{x}, \forall i \in I, \forall k \in K \\
& \tau_{i k} \mathbf{e}+\rho(\mathbf{x}-\mathbf{e}) \leq \mathbf{d}_{i k}, \forall i \in I, \forall k \in K \\
& \mathbf{d}_{i k} \leq \tau_{i k} \mathbf{e}+\rho(\mathbf{e}-\mathbf{x}), \forall i \in I, \forall k \in K \\
& \tau_{i k} \geq 0, \forall i \in I, \forall k \in K \\
& q_{i k} \leq x_{i}, \forall i \in I, \forall k \in K \\
& x_{i} \in\{0,1\}, \forall i \in I .
\end{aligned}
$$

From Appendix B.1, we note that constraints (28)-(31) are imposed to linearize the term $\tau_{i k} \mathbf{x}$. Let us temporarily restore the nonlinear term $\tau_{i k} \mathbf{x}$, and the constraints (25)-(32) become:

$$
\begin{aligned}
& \left\langle\mathbf{M}_{i k}, \boldsymbol{\Sigma}_{i k}\right\rangle \leq 1-q_{i k}, \forall i \in I, \forall k \in K \\
& \mathbf{M}_{i k} \succeq 0, \forall i \in I, \forall k \in K \\
& \mathbf{M}_{i k}+\left[\begin{array}{cc}
0 & \tau_{i k} \mathbf{x} \\
\tau_{i k} \mathbf{x}^{T}-1-2 \tau_{i k} b_{k}
\end{array}\right] \succeq 0, \forall i \in I, \forall k \in K \\
& \tau_{i k} \geq 0, \forall i \in I, \forall k \in K .
\end{aligned}
$$

Moreover, it can be shown that $\tau_{i k}$ in the optimal solution is strictly positive (as long as an optimal solution exists), as $\tau_{i k}=0$ leads to $q_{i k} \leq 0$. We are then able to rewrite the above constraints as follows, by defining new variables $\tilde{\tau}_{i k}=\frac{1}{\tau_{i k}} \geq 0$ and $\tilde{\mathbf{M}}_{i k}=\mathbf{M}_{i k} \tilde{\tau}_{i k}$ :

$$
\begin{aligned}
& \left\langle\tilde{\mathbf{M}}_{i k}, \boldsymbol{\Sigma}_{i k}\right\rangle \leq \tilde{\tau}_{i k}\left(1-q_{i k}\right), \forall i \in I, \forall k \in K \\
& \tilde{\mathbf{M}}_{i k} \succeq 0, \forall i \in I, \forall k \in K \\
& \tilde{\mathbf{M}}_{i k}+\left[\begin{array}{c}
0 \quad \mathbf{x} \\
\mathbf{x}^{T}-\tilde{\tau}_{i k}-2 b_{k}
\end{array}\right] \succeq 0, \forall i \in I, \forall k \in K \\
& \tilde{\tau}_{i k} \geq 0, \forall i \in I, \forall k \in K
\end{aligned}
$$

We now show that constraints (33)-(36) can be reformulated in second-order conic form. Let $\mathcal{P}$ be the set of probability distributions with mean $\mu$ and covariance matrix $\boldsymbol{\Gamma} \succ 0$. Let $\epsilon \in(0,1]$ and $\gamma \in \mathbb{R}$ given. The following propositions are equivalent (El Ghaoui et al. 2003): 
1. $\sqrt{\frac{1-\epsilon}{\epsilon}} \sqrt{\mathbf{x}^{T} \boldsymbol{\Gamma}_{i} \mathbf{x}}-\mu^{T} \mathbf{x} \leq \gamma$

2. There exist a symmetric matrix $\mathbf{M}$ and $\tau \in \mathbb{R}$ such that

$$
\begin{aligned}
& \langle\mathbf{M}, \mathbf{\Sigma}\rangle \leq \tau \epsilon \\
& \mathbf{M} \succeq 0 \\
& \mathbf{M}+\left[\begin{array}{cc}
0 & \mathbf{x} \\
\mathbf{x}^{T} & -\tau+2 \gamma
\end{array}\right] \succeq 0 \\
& \tau \geq 0
\end{aligned}
$$

where $\boldsymbol{\Sigma}$ is the second-moment matrix.

By viewing $1-q_{i k}$ as $\epsilon$ and $-b_{k}$ as $\gamma$ in the above result, the semidefinite constraint (33)-(36) can be expressed as

$$
\begin{aligned}
& \sqrt{\frac{q_{i k}}{1-q_{i k}}} \sqrt{X^{T} \boldsymbol{\Gamma}_{i} X}-\overline{\mathbf{a}}_{i}^{T} \mathbf{x}+b_{k} \leq 0 \\
& \equiv\left\{\begin{array}{l}
1-q_{i k} \geq \frac{\mathbf{x}^{T} \boldsymbol{\Gamma}_{i} \mathbf{x}}{\left(\overline{\mathbf{a}}_{i}^{T} \mathbf{x}-b_{k}\right)^{2}+\mathbf{x}^{T} \Gamma_{i} \mathbf{x}} \\
\overline{\mathbf{a}}_{i}^{T} \mathbf{x}-b_{k} \geq 0 .
\end{array}\right.
\end{aligned}
$$

In the case of $\overline{\mathbf{a}}_{i}^{T} \mathbf{x}-b_{k} \leq 0$, the worst case probability is 1 , because there exists a feasible two-point distribution of $\overline{\mathbf{a}}_{i}^{T} \mathbf{x}$ with both points less than $b_{k}$. To allow such possibility, we further introduce a set of disjunctive constraints. When $\overline{\mathbf{a}}_{i}^{T} \mathbf{x}-b_{k} \leq 0$, we set $q_{i k}$ to 0 . Therefore, we have either $\overline{\mathbf{a}}_{i}^{T} \mathbf{x} \geq b_{k}$ or $q_{i k}=0$. By introducing new variables, we can express the disjunctive constraints as the feasible set:

$$
\mathcal{X}_{i k}=\left\{\left(\mathbf{x}, q_{i k}\right): \begin{array}{l}
b_{k} u_{i k} \leq \overline{\mathbf{a}}_{i}^{T} \mathbf{x} \\
q_{i k} \leq u_{i k} \\
u_{i k} \in\{0,1\}
\end{array}\right\}
$$

Moreover, we linearize the term $x_{j_{1}} x_{j_{2}}$ with $z_{j_{1} j_{2}}$ defined in

$$
\mathcal{Z}=\left\{\left(z_{j_{1} j_{2}}, x_{j_{1}}, x_{j_{2}}\right): \begin{array}{l}
z_{j_{1} j_{2}} \leq x_{j_{1}} \\
z_{j_{1} j_{2}} \leq x_{j_{2}} \\
x_{j_{1}}+x_{j_{2}}-1 \leq z_{j_{1} j_{2}} \\
z_{j_{1} j_{2}} \geq 0
\end{array}\right\} .
$$

Lastly, the first inequality in constraint (37) can be expressed as $\left(1-q_{i k}+\left(\overline{\mathbf{a}}_{i}^{T} \mathbf{x}-b_{k}\right)^{2}+\mathbf{x}^{T} \boldsymbol{\Gamma}_{i} \mathbf{x}\right)^{2} \geq$ $4 \mathbf{x}^{T} \boldsymbol{\Gamma}_{i} \mathbf{x}+\left(1-q_{i k}-\left(\overline{\mathbf{a}}_{i}^{T} \mathbf{x}-b_{k}\right)^{2}-\mathbf{x}^{T} \boldsymbol{\Gamma}_{i} \mathbf{x}\right)^{2}$. Since $z_{j_{1} j_{2}}=x_{j_{1}} x_{j_{2}}$ in (39), we further linearize the terms $\left(\overline{\mathbf{a}}_{i}^{T} \mathbf{x}\right)^{2}$ and $\mathbf{x}^{T} \boldsymbol{\Gamma}_{i} \mathbf{x}$ as $\sum_{\left(j_{1}, j_{2}\right) \in I \times I} \bar{a}_{i j_{1}} \bar{a}_{i j_{2}} z_{j_{1} j_{2}}$ and $\sum_{\left(j_{1}, j_{2}\right) \in I \times I} \sigma_{i j_{1} j_{2}} z_{j_{1} j_{2}}$ respectively. Let $v_{i k}=b_{k}^{2}+$ $\sum_{\left(j_{1}, j_{2}\right) \in I \times I}\left(\bar{a}_{i j_{1}} \bar{a}_{i j_{2}}+\sigma_{i j_{1} j_{2}}\right) z_{j_{1} j_{2}}-2 b_{k} \sum_{j \in I} \bar{a}_{i j} x_{j}$, it is reorganized as $\left\|\begin{array}{c}1-q_{i k}-v_{i k} \\ 2 \boldsymbol{\Gamma}_{i}^{\frac{1}{2}} \mathbf{x}\end{array}\right\|_{2} \leq 1-q_{i k}+v_{i k}$ and leads to the second-order cone constraint (5).

\section{B.3. Detailed Formulation of the MISOCP (22)}

$$
\begin{aligned}
& \max _{\substack{x_{i}, q_{i k}, N, \Psi_{i j} \\
\psi_{i j}, \psi_{i j}^{\prime}, \phi_{i j}, \lambda_{i}}} \sum_{i \in I} \sum_{k \in K} f Q_{i k} q_{i k}-\sum_{i \in I} g_{i} x_{i}+\xi\left(\sum_{j \in I} \sum_{i \in I} r t_{i j} \psi_{i j}-\sum_{i \in I} \sum_{j \in I} \eta \tau_{i j} \phi_{i j}-\sum_{i \in I} c \lambda_{i}\right)-h N \\
& \text { s.t. } \\
& \qquad \begin{array}{l}
1-q_{i k}-v_{i k} \\
2 \Gamma_{i}^{\frac{1}{2}} \mathbf{x}
\end{array} \|_{2} \leq 1-q_{i k}+v_{i k}, \forall i \in I, \forall k \in K \\
& v_{i k}=b_{k}^{2}+\sum_{\left(j_{1}, j_{2}\right) \in I \times I}\left(\bar{a}_{i j_{1}} \bar{a}_{i j_{2}}+\sigma_{i j_{1} j_{2}}\right) z_{j_{1} j_{2}}-2 b_{k} \sum_{j \in I} \bar{a}_{i j} x_{j}, \forall i \in I, \forall k \in K
\end{aligned}
$$




$$
\begin{aligned}
& q_{i k} \leq x_{i}, \forall i \in I, \forall k \in K \\
& \Psi_{i j}=P_{i j} \mu_{i} \sum_{k \in K} w_{i k} q_{i k}, \forall i, j \in I \\
& \psi_{i j}^{\prime} \geq \alpha \Psi_{i j}, \forall i, j \in I \\
& \psi_{i j}^{\prime} \leq \Psi_{i j}, \forall i, j \in I \\
& \psi_{i j}^{\prime}=P_{i j} \sum_{l \in I} \psi_{i l}^{\prime}, \forall i, j \in I \\
& \psi_{i j} \leq \psi_{i j}^{\prime}, \forall i, j \in I \\
& \psi_{i j} \leq P_{i j} \mu_{i} x_{j}, \forall i, j \in I \\
& \psi_{i j}^{\prime}+P_{i j} \mu_{i}\left(x_{j}-1\right) \leq \psi_{i j}, \forall i, j \in I \\
& \sum_{j} \psi_{j i}+\sum_{j} \phi_{j i}=\sum_{j} \psi_{i j}+\sum_{j} \phi_{i j}, \forall i \in I \\
& \lambda_{i}=P_{c} \sum_{j \in J} \psi_{j i}, \forall i \in I \\
& \sum_{i \in I} \frac{\alpha}{1-\alpha} x_{i}+\sum_{j \in I} \sum_{i \in I} t_{i j} \psi_{i j}+\sum_{i \in I} \sum_{j \in I} \tau_{i j} \phi_{i j}+\sum_{i \in I} t_{c} \lambda_{i} \leq N \\
& \left(z_{j_{1} j_{2}}, x_{j_{1}}, x_{j_{2}}\right) \in \mathcal{Z}, \forall j_{1}, j_{2} \in I \\
& \left(\mathbf{x}, q_{i k}\right) \in \mathcal{X}_{i k}, \forall i \in I, \forall k \in K \\
& N, q_{i k}, \psi_{i j}, \psi_{i j}^{\prime}, \Psi_{i j}, \phi_{i j}, \lambda_{i} \geq 0, \forall i, j \in I, k \in K \\
& x_{i} \in\{0,1\}, \forall i \in I .
\end{aligned}
$$

\section{B.4. Time-varying Travel Pattern}

The customer adoption decision depends only on the service region coverage and thus the constraints (3) and (5)-(7) from the adoption rate model still hold. To deal with time-varying travel patterns, we partition the day into $T$ periods (indexed by $t$ ), within each of which the travel pattern is assumed (approximated) to be stationary, i.e., represented by $P_{i j}^{t}$ and $\mu_{i}^{t}$ for each period $t$. Let $l(t)$ be the length of period $t$, the problem in the presence of time-varying travel patterns, following the pointwise stationary approximation (Green and Kolesar 1991), is then formulated as:

$$
\max _{\substack{x_{i}, q_{i k}, N, \Psi_{i j}^{t} \\ \psi_{i j}^{t}, \psi_{i j}^{\prime}, \phi_{i j}^{t}, \lambda_{i}^{t}}} \sum_{i \in I} \sum_{k \in K} f Q_{i k} q_{i k}-\sum_{i \in I} g_{i} x_{i}+\xi \sum_{t \in T} l(t)\left(\sum_{j \in I} \sum_{i \in I} r t_{i j} \psi_{i j}^{t}-\sum_{i \in I} \sum_{j \in I} \eta \tau_{i j} \phi_{i j}^{t}-\sum_{i \in I} c \lambda_{i}^{t}\right)-h N
$$

s.t.

$$
\begin{aligned}
& \text { Constraint }(3) \&(5)-(7) \\
& \Psi_{i j}^{t}=P_{i j}^{t} \mu_{i}^{t} \sum_{k \in K} w_{i k} q_{i k}, \forall i, j \in I, t \in T \\
& \psi_{i j}^{\prime t} \geq \alpha \Psi_{i j}^{t}, \forall i \in I, t \in T \\
& \psi_{i j}^{\prime} \leq \Psi_{i j}^{t}, \forall i, j \in I, t \in T \\
& \psi_{i j}^{\prime t}=P_{i j}^{t} \sum_{l \in I} \psi_{i l}^{\prime}, \forall i, j \in I, t \in T \\
& \psi_{i j}^{t} \leq \psi_{i j}^{\prime}, \forall i, j \in I, t \in T
\end{aligned}
$$




$$
\begin{aligned}
& \psi_{i j}^{t} \leq P_{i j}^{t} \mu_{i}^{t} x_{j}, \forall i, j \in I, t \in T \\
& \psi_{i j}^{\prime}+P_{i j}^{t} \mu_{i}^{t}\left(x_{j}-1\right) \leq \psi_{i j}^{t}, \forall i, j \in I, t \in T \\
& \sum_{j} \psi_{j i}^{t}+\sum_{j} \phi_{j i}^{t}=\sum_{j} \psi_{i j}^{t}+\sum_{j} \phi_{i j}^{t}, \forall i \in I, t \in T \\
& \lambda_{i}^{t}=P_{c} \sum_{j \in J} \psi_{j i}^{t}, \forall i \in I, t \in T \\
& \sum_{i \in I} \frac{\alpha}{1-\alpha} x_{i}+\sum_{j \in I} \sum_{i \in I} t_{i j} \frac{\psi_{i j}^{t}}{l(t)}+\sum_{i \in I} \sum_{j \in I} \tau_{i j} \frac{\phi_{i j}^{t}}{l(t)}+\sum_{i \in I} t_{c} \frac{\lambda_{i}^{t}}{l(t)} \leq N, \forall t \in T \\
& N, q_{i k}, \psi_{i j}^{t}, \psi_{i j}^{\prime}, \Psi_{i j}^{t}, \phi_{i j}^{t}, \lambda_{i}^{t} \geq 0, \forall i, j \in I, \forall k \in K, \forall t \in T \\
& x_{i} \in\{0,1\}, \forall i \in I .
\end{aligned}
$$

\section{B.5. Proof of Proposition 2}

Given a service region design $I^{\prime} \subseteq I$, let $q_{i k}^{\prime}$ be the realized adoption rate, e.g. $q_{i k}^{\prime}=\operatorname{Prob}\left(\sum_{j \in I^{\prime}} a_{i j} x_{j} \geq b_{k}\right)$. Note that the fleet size constraint (21) is binding, the formulation (22) becomes a linear program below:

$$
\begin{aligned}
\max _{q_{i k}, \psi_{i j}, \phi_{i j}} & \sum_{i \in I^{\prime}} \sum_{k \in K} f Q_{i k} q_{i k}-\sum_{i \in I^{\prime}} g_{i} x_{i}+\xi\left(\sum_{j \in I^{\prime}} \sum_{i \in I^{\prime}} r t_{i j} \psi_{i j}-\sum_{i \in I^{\prime}} \sum_{j \in I^{\prime}} \eta \tau_{i j} \phi_{i j}-\sum_{i \in I^{\prime}} c P_{c} \sum_{j \in I^{\prime}} \psi_{j i}\right) \\
& -h\left(\sum_{i \in I^{\prime}} \frac{\alpha}{1-\alpha} x_{i}+\sum_{j \in I^{\prime}} \sum_{i \in I^{\prime}} t_{i j} \psi_{i j}+\sum_{i \in I^{\prime}} \sum_{j \in I^{\prime}} \tau_{i j} \phi_{i j}+\sum_{i \in I^{\prime}} t_{c} P_{c} \sum_{j \in I^{\prime}} \psi_{j i}\right) \\
\text { s.t. } & \\
& q_{i k} \leq q_{i k}^{\prime}, \forall i \in I^{\prime}, \forall k \in K \\
& \psi_{i j} \geq \alpha P_{i j} \mu_{i} \sum_{k \in K} w_{i k} q_{i k}, \forall i \in I^{\prime} \\
& \psi_{i j} \leq P_{i j} \mu_{i} \sum_{k \in K} w_{i k} q_{i k}, \forall i, j \in I^{\prime} \\
& \psi_{i j}=P_{i j} \sum_{l \in I^{\prime}} \psi_{i l}, \forall i, j \in I^{\prime} \\
& \sum_{j \in I^{\prime}} \psi_{j i}+\sum_{j \in I^{\prime}} \phi_{j i}=\sum_{j \in I^{\prime}} \psi_{i j}+\sum_{j \in I^{\prime}} \phi_{i j}, \forall i \in I^{\prime} \\
& q_{i k}, \psi_{i j}, \phi_{i j} \geq 0, \forall i, j \in I^{\prime}, \forall k \in K .
\end{aligned}
$$

Since the service region design $I^{\prime}$ is given, the terms with $x_{i}$ are constant and can be omitted. We define an equivalent objective function

$$
\begin{aligned}
\mathbf{H}\left(q_{i k}, \psi_{i j}, \phi_{i j}\right) & =\sum_{i \in I^{\prime}} \sum_{k \in K} f Q_{i k} q_{i k}+\sum_{j \in I^{\prime}} \sum_{i \in I^{\prime}}\left[(\xi r-h) t_{i j}-P_{c}\left(\xi c+h t_{c}\right)\right] \psi_{i j}-\sum_{j \in I^{\prime}} \sum_{i \in I^{\prime}}(\xi \eta+h) \tau_{i j} \phi_{i j} \\
& =\sum_{i \in I^{\prime}} \sum_{k \in K} f Q_{i k} q_{i k}+\sum_{j \in I^{\prime}} \sum_{i \in I^{\prime}}\left(u_{i j} \psi_{i j}-v_{i j} \phi_{i j}\right)
\end{aligned}
$$

where $u_{i j}=(\xi r-h) t_{i j}-P_{c}\left(\xi c+h t_{c}\right)$ and $v_{i j}=(\xi \eta+h) \tau_{i j}$.

Let $y_{i k}, m_{i j}, n_{i j}, s_{i j}$ and $z_{i}$ be the dual variables. We write the dual formulation of the above linear program as

$$
\min _{y_{i k}, m_{i j}, n_{i j}, s_{i j}, z_{i}} \sum_{i \in I^{\prime}} \sum_{k \in K} q_{i k}^{\prime} y_{i k}
$$




$$
\begin{aligned}
& \text { s.t. } \\
& \begin{array}{l}
f Q_{i k}+\sum_{j \in I^{\prime}}\left(m_{i j}-\alpha n_{i j}\right) P_{i j} \mu_{i} w_{i k} \leq y_{i k}, \forall i \in I^{\prime}, \forall k \in K \\
u_{i j}-m_{i j}+n_{i j}+\sum_{l \in I^{\prime}} P_{i l} s_{i l}-s_{i j}+z_{j}-z_{i} \leq 0, \forall i, j \in I^{\prime} \\
-v_{j i} \leq z_{j}-z_{i}, \forall i, j \in I^{\prime} \\
\\
y_{i k}, m_{i j}, n_{i j} \geq 0, \forall i, j \in I^{\prime}
\end{array}
\end{aligned}
$$

From inequalities (41) and (42), we have

$$
\begin{aligned}
& u_{i j}-v_{j i}+\sum_{l \in I^{\prime}} P_{i l} s_{i l}-s_{i j} \leq m_{i j}-n_{i j} \leq m_{i j}-\alpha n_{i j}, \forall i, j \in I^{\prime} \\
& \Rightarrow \sum_{j \in I^{\prime}} P_{i j}\left(u_{i j}-v_{j i}\right) \leq \sum_{j \in I^{\prime}} P_{i j}\left(m_{i j}-\alpha n_{i j}\right), \forall i \in I^{\prime}
\end{aligned}
$$

This inequality provides a lower bound to the LHS of constraint (40). That is,

$$
f Q_{i k}+\mu_{i} w_{i k} \sum_{j \in I^{\prime}} P_{i j}\left(u_{i j}-v_{j i}\right) \leq y_{i k}, \forall i \in I^{\prime}, \forall k \in K
$$

To have the adoption rate constraint $q_{i k} \leq q_{i k}^{\prime}$ tight, we need the associated dual variable $y_{i k}>0$. Therefore, by noting that $v_{i i}=0$, the corresponding sufficient condition is to have

$$
f Q_{i k}+\mu_{i} w_{i k} P_{i i} u_{i i}+\mu_{i} w_{i k} \sum_{\substack{j \in I^{\prime} \\ j \neq i}} P_{i j}\left(u_{i j}-v_{j i}\right)>0, \forall i \in I^{\prime}, \forall k \in K .
$$

\section{Appendix C: Estimation of Key Parameters}

\section{C.1. Utility Parameters}

We use the trip distributions that describe customer preferences over destinations as proxies to utility parameters a. Our study focuses on the 61 candidate zip codes in San Diego County, excluding remote and military areas. We estimate the origin-destination trip distributions for the 61 candidate zip codes by extrapolating, using a gravity model, from the Car2Go operations data for the 18 zip codes they currently cover. The sample daily trips for each origin-destination pair of the 18 zip codes are counted and the outbound (inbound) trips from (to) each zip code are summarized in Table 4.

Table 4 Sample Daily Outbound(Inbound) Trips in Current Car2Go Service Region

\begin{tabular}{|c|c|c|c|c|c|}
\hline $\mathbf{9 1 9 1 0}$ & $\mathbf{9 1 9 1 1}$ & $\mathbf{9 2 1 0 1}$ & $\mathbf{9 2 1 0 2}$ & $\mathbf{9 2 1 0 3}$ & $\mathbf{9 2 1 0 4}$ \\
\hline $7.92(8.19)$ & $3.19(3.27)$ & $334.04(335.23)$ & $55.69(54.77)$ & $144.04(144.04)$ & $81.69(80.5)$ \\
\hline $\mathbf{9 2 1 0 5}$ & $\mathbf{9 2 1 0 6}$ & $\mathbf{9 2 1 0 7}$ & $\mathbf{9 2 1 0 8}$ & $\mathbf{9 2 1 0 9}$ & $\mathbf{9 2 1 1 0}$ \\
\hline $11.23(10.35)$ & $45.89(45.65)$ & $54.23(54.92)$ & $50.77(51.92)$ & $84.08(83.96)$ & $44.92(46)$ \\
\hline $\mathbf{9 2 1 1 1}$ & $\mathbf{9 2 1 1 3}$ & $\mathbf{9 2 1 1 5}$ & $\mathbf{9 2 1 1 6}$ & $\mathbf{9 2 1 2 0}$ & $\mathbf{9 2 1 2 3}$ \\
\hline $0.35(0.35)$ & $1.23(1.19)$ & $14.62(14.85)$ & $60.08(58.73)$ & $1.12(1.19)$ & $0.12(0.08)$ \\
\hline
\end{tabular}

Table 4 suggests a large variation in trip demands of the 18 zip codes. The majority of the trips were generated in the downtown San Diego with zip codes 92101 and 92103, while few trip demands are observed 
from zip codes 92111 and 92123 . To better capture the demand pattern, we exclude zip codes with very low demands, e.g., 91911, 92111, 92113, 92120 and 92123, in the estimation of the gravity model, since inclusion of these outliers led to significantly worse fits.

We first fit a gravity model for the sample trip rates for one-way trips among 18 zip codes (i.e., trips where origins and destinations are in distinct zip codes). Trips that originate and end within the same zip code are handled later. Besides the (working-age) population factor in the classic gravity model, we also test other socioeconomic factors, such as per capita income, business establishments, students enrollments and workplace population, that may affect the trip distributions. The only statistical significant factors we find are working-age population and per capita income. Similarly, Wills (1986) integrates income as a destination-attribute variable in his trip distribution models. Hence, we fit the gravity model for one-way trips as follows:

$$
T_{i j}=\beta_{1} \frac{\operatorname{Pop}_{i} \operatorname{Pop}_{j} \operatorname{Inc} c_{i}^{\beta_{2}} \operatorname{Inc} c_{j}^{\beta_{3}}}{\operatorname{dist}_{i j}^{\beta_{4}}}
$$

where $P o p_{i}$ is the working-age population and $I n c_{i}$ is the per capita income in $i$. The number of trips generated from $i$ is proportional to $P o p_{i}$ while $P o p_{j}$ and $I n c_{j}$ are indicators of the attractiveness of destination $j$.

Let $\hat{T}_{i j}^{C a r 2 G o}$ be the observed trips from Car2Go's operations data from $i$ to $j$. The sample daily trip are adjusted by the current adoption rate (current regular members divided by market size in the current service region), e.g., $\hat{T}_{i j}^{\text {Car2Go } /(C u r r e n t ~ A d o p t i o n ~ R a t e) . ~ W e ~ t h e n ~ a p p l y ~ l o g-l i n e a r ~ r e g r e s s i o n ~ t o ~} \hat{T}_{i j}$ and obtain the gravity model in (43) for the aggregated daily trip distribution below with adjusted R-squared of 0.7515 and residual standard error of 0.9227 .

$$
T_{i j}=\exp (-62.212) \frac{\text { Pop }_{i} \text { Pop }_{j} \operatorname{Inc}_{i}^{2.253} \operatorname{Inc}_{j}^{2.249}}{\operatorname{dist}_{i j}^{2.013}} .
$$

For trips that begin and end within the same zip code (round trips), we fit the following model using the same socialeconomic factors as independent variables:

$$
T_{i i}=\beta_{1} \operatorname{Pop}_{i} \operatorname{Inc} c_{i}^{\beta_{2}} .
$$

The fitted model, as shown below, has adjusted R-squared of 0.7845 and residual standard error:

$$
T_{i i}=\exp (-43.194) \text { Pop }_{i} \operatorname{Inc}_{i}^{3.413} \text {. }
$$

With the residual standard errors provided by the regressions, we randomly generate 1000 sample trip distributions for the 61 candidate zip codes using gravity models in (44) and (45). In each sample $n$, the sample utility parameter $\hat{a}_{i j}^{n}$ is estimated by normalizing the outbound trips by their destinations:

$$
\hat{a}_{i j}^{n}=\frac{\hat{T}_{i j}^{n}}{\sum_{j \in I} \hat{T}_{i j}^{n}}
$$

The mean of utility parameter, $\bar{a}_{i j}$, is then calculated as the sample average of $\hat{a}_{i j}^{n}$. We also construct the estimated diagonal covariance matrix $\Gamma_{i}$ for each $i$ with $\sigma_{i j}^{2}$ set to be sample variance of $\hat{a}_{i j}^{n}$ for all origin $i$ and destination $j$.

Lastly, the outbound demand rate $\mu_{i}$ is estimated by averaging the total outbound trips $\hat{\mu}_{i}^{n}=\sum_{j \in I} \hat{T}_{i j}^{n}$ over all samples. The average travel speed in San Diego is assumed to be $31 \mathrm{mph}$ (John Kleint 2016) for conversion of travel time to travel distance as $d_{i j}(\operatorname{mile})=t_{i j}(\min ) \times \frac{31(\mathrm{mph})}{60(\min / \mathrm{h})}$. 


\section{C.2. Adoption Requirement}

The aspirational level $b$ is evaluated based on the mode choice data in CHTS. We summarize the mode choice distribution in 4 categories: non-moto (including walking and cycling, etc.), private car (including driver, passenger, car rental and carpool, etc,.), bus (including bus and shuttle, etc.), and rail (including subways and light rail, etc.). We focus on motorized trips, and group bus and rail modes to public transportation. Using K-means clustering approach (e.g., Hartigan and Wong (1979)), we group the individuals into 3 clusters with different mode choice distributions as shown in Table 5.

\section{Table 5 Mode Choice Distribution}

\begin{tabular}{|c|c|c|}
\hline Group & Car & Public \\
\hline 1 & 0.01 & 0.99 \\
2 & 0.53 & 0.47 \\
3 & 1.00 & 0.00 \\
\hline
\end{tabular}

For instance, the potential travelers from Group 2 choose to drive for $53 \%$ of their trips. We hence set $b=53 \%$ for these customers, i.e., they would be willing to switch to car sharing if it covers at least $53 \%$ of their travel needs. After adoption, customers will use car sharing service regularly with destinations that are in the service region. Following the same reasoning, Groups 1 and 3 are not target segments for Car2Go because these customers either never drive or rely too much on their own cars that they would require $100 \%$ service coverage. As a result, the target Group 2 account for $4.05 \%$ of the entire population and we set $Q_{i k}=20 \% \times 4.05 \% \times P o p_{i}$, where $P o p_{i}$ is the population in area $i$, by assuming $20 \%$ local market share in car sharing business. Due to the limited sample sizes at zip code level, we assume all zip codes share the same mode choice distribution.

\section{C.3. Coverage Costs}

The fixed coverage cost associated with serving region $i$ includes investments in charging infrastructure, in partnership with charging service providers. As planned in 2011, Car2Go's fleet in San Diego can be recharged at 1000 Blink EV charging stations (Engadget 2014). We use the EV charging station data from U.S. Department of Energy (2014a) to estimate the number of chargers required in each zip code to support the EV sharing system. For each zip code in the current service region, we compute the charger density (CD) by dividing the number of chargers (CG) with the land area (LA). From linear regression with various socialeconomic factors, we find that number of business establishment (BE) is the only significant factor to CD with the fitted model as below

$$
C D_{i}=3.19 \exp (-11) B E_{i}
$$

The number of chargers needed for each candidate area is then approximated by

$$
C G_{i}=\max \left\{C D_{i}, 0\right\} \times L A_{i} .
$$

Suppose the investment in each charger by Car2Go through partnership with Blink is $h_{c}$ (e.g., $\$ 800$, about half of the typical cost to install a household Level 2 charger in California (California Plug-in Electric Vehicle Collaborative 2016)), then the coverage cost for region $i$ follows $g_{i}=h_{c} C G_{i}$. 


\section{Appendix D: Heuristic Formulation}

\section{D.1. H1 Formulation}

The heuristic $H_{1}$ solves the following mixed integer program based on a stylized view on adoption behavior (assuming $q_{s}=b$ ):

$$
\begin{aligned}
& \max _{\substack{x_{i}, N, \Psi_{i j} \\
\psi_{i j}, \psi_{i j}^{\prime}, \phi_{i j}, \lambda_{i}}} \sum_{i \in I} f Q_{i} q_{s}-\sum_{i \in I} g_{i} x_{i}+\xi\left(\sum_{j \in I} \sum_{i \in I} r t_{i j} \psi_{i j}-\sum_{i \in I} \sum_{j \in I} \eta \tau_{i j} \phi_{i j}-\sum_{i \in I} c \lambda_{i}\right)-h N \\
& \text { s.t. } \\
& \quad \text { Constraint }(9)-(21) \\
& \Psi_{i j}=\mu_{i} P_{i j} q_{s}, \forall i \in I, j \in J \\
& N, \psi_{i j}, \psi_{i j}^{\prime}, \Psi_{i j}, \phi_{i j}, \lambda_{i} \geq 0 \\
& x_{i} \in\{0,1\}, \forall i \in I .
\end{aligned}
$$

\section{D.2. H2 Formulation}

The heuristic $H_{2}$ solves the following mixed integer SOCP formulated based on a stylized view on fleet operations:

$$
\begin{aligned}
\max _{\substack{x_{i}, q_{i}, N, \Psi_{i j} \\
\psi_{i j}, \psi_{i j}^{\prime}, \phi_{i j}, \lambda_{i} \\
\text { s.t. }}} & \sum_{i \in I} f Q_{i} q_{i}-\sum_{i \in I} g_{i} x_{i}+\xi\left(\sum_{j \in I} \sum_{i \in I} r t_{i j} \psi_{i j}-\sum_{i \in I} \sum_{j \in I} \eta \tau_{i j} \phi_{i j}-\sum_{i \in I} c \lambda_{i}\right)-h N \\
& \text { Constraint }(3) \&(5)-(19) \\
& N=\bar{t} \times \sum_{i \in I} x_{i} \mu_{i} q_{i} \\
& N, \psi_{i j}, \psi_{i j}^{\prime}, \Psi_{i j}, \phi_{i j}, \lambda_{i} \geq 0 \\
& x_{i} \in\{0,1\}, \forall i \in I .
\end{aligned}
$$

\section{D.3. H3 Formulation}

The heuristic $H_{3}$ solves the following mixed integer program formulated based on a stylized view on both adoption behavior and fleet operations:

$$
\max _{\substack{x_{i}, N, \Psi_{i j} \\ \psi_{i j}, \psi_{i j}, \phi_{i j}, \lambda_{i}}} \sum_{i \in I} f Q_{i} q_{s}-\sum_{i \in I} g_{i} x_{i}+\xi\left(\sum_{j \in I} \sum_{i \in I} r t_{i j} \psi_{i j}-\sum_{i \in I} \sum_{j \in I} \eta \tau_{i j} \phi_{i j}-\sum_{i \in I} c \lambda_{i}\right)-h N
$$

s.t.

Constraint (9) - (19)

$$
\begin{aligned}
& \Psi_{i j}=\mu_{i} P_{i j} q_{s}, \forall i \in I, j \in J \\
& N=\bar{t} \times \sum_{i \in I} x_{i} \mu_{i} q_{i} \\
& N, \psi_{i j}, \psi_{i j}^{\prime}, \Psi_{i j}, \phi_{i j}, \lambda_{i} \geq 0 \\
& x_{i} \in\{0,1\}, \forall i \in I .
\end{aligned}
$$

